\title{
A Review of Fetal ECG Signal Processing Issues and Promising Directions
}

\author{
Reza Sameni ${ }^{1, *}$ and Gari D. Clifford ${ }^{2,3}$ \\ ${ }^{1}$ School of Electrical \& Computer Engineering, Shiraz University, Shiraz, Iran \\ ${ }^{2}$ Institute of Biomedical Engineering, Department of Engineering Science, University of Oxford, UK \\ ${ }^{3}$ Division of Sleep Medicine, Department of Medicine, Harvard University, Boston, USA
}

\begin{abstract}
The field of electrocardiography has been in existence for over a century, yet despite significant advances in adult clinical electrocardiography, signal processing techniques and fast digital processors, the analysis of fetal ECGs is still in its infancy. This is, partly due to a lack of availability of gold standard databases, partly due to the relatively low signal-to-noise ratio of the fetal ECG compared to the maternal ECG (caused by the various media between the fetal heart and the measuring electrodes, and the fact that the fetal heart is simply smaller), and in part, due to the less complete clinical knowledge concerning fetal cardiac function and development. In this paper we review a range of promising recording and signal processing techniques for fetal ECG analysis that have been developed over the last forty years, and discuss both their shortcomings and advantages. Before doing so, however, we review fetal cardiac development, and the etiology of the fetal ECG. A selection of relevant models for the fetal/maternal ECG mixture is also discussed. In light of current understanding of the fetal ECG, we then attempt to justify recommendations for promising future directions in signal processing, and database creation.
\end{abstract}

Keywords: Fetal electrocardiogram, source separation, signal processing, adaptive filtering.

\section{INTRODUCTION}

Heart defects are among the most common birth defects and the leading cause of birth defect-related deaths [1, 2]. Every year, about one out of 125 babies are born with some form of congenital heart defects [3]. The defect may be so slight that the baby appears healthy for many years after birth, or so severe that its life is in immediate danger. Congenital heart defects originate in early stages of pregnancy when the heart is forming and they can affect any of the parts or functions of the heart. Cardiac anomalies may occur due to a genetic syndrome, inherited disorder, or environmental factors such as infections or drug misuse [1, 4]. However, except for during labor, fetal electrocardiography has not proved an effective tool for imaging specific structural defects. Rather, fetal electrocardiography has been confined to more global issues such as general ischemia due to specific fetal positioning that chokes the umbilical cord [5]. The reason for this limitation is that the noninvasive fetal electrocardiogram (ECG) is contaminated by fetal brain activity, myographic (muscle) signals (from both the mother and fetus), movement artifacts and multiple layers of different dielectric biological media through which the electrical signals must pass.

When continuous electronic fetal heart rate monitoring was introduced into clinical practice in the 1970s, there was enormous optimism that the widespread use of this

*Address correspondence to this author at the School of Electrical \& Computer Engineering, Shiraz University, Shiraz, Iran;

Tel: +98 7116133169 ; Fax: +98 71164746 05;

E-mails: rsameni@shirazu.ac.ir, gari@robots.ox.ac.uk technology would reduce the incidence of intra-partum fetal injury and death. Unfortunately, fetal heart rate monitoring has not lived up to its initial promise. A meta-analysis of nine randomized, controlled trials comparing fetal monitoring to intermittent auscultation of the fetal heart rate showed that current monitoring techniques increase the use of cesarean, forceps, and vacuum delivery, but do not reduce perinatal morbidity or mortality [6].

Since the advent of fetal heart rate monitoring 40 years ago, there have been no clinically significant advances in intra-partum fetal monitoring. Moreover, continuous fetal monitoring is utilized in over $85 \%$ of labor episodes in the United States, and represents the standard of care [7]. Fetal monitoring today is based entirely on the fetal heart rate and does not incorporate characteristics of the fetal ECG (fECG) waveform characteristics that are the cornerstone of cardiac evaluation of both children and adults. The primary reason for the exclusion of this most critical source of information from clinical practice is that the technology to reliably measure fECG is largely unavailable. As a consequence, research correlating ECG characteristics to neonatal outcomes has not been done on a large scale.

Most cardiac defects have some manifestation in the morphology of cardiac electrical signals, which are recorded by electrocardiography and are believed to contain much more information as compared with conventional sonographic methods [8]. However, no signal processing technique has been able to reliably deliver an undistorted fECG signal from electrodes placed on the maternal abdomen because of the low signal-to-noise ration (SNR) of the fECG recorded from the maternal body surface. The application of 
fetal electrocardiography has therefore been almost completely limited to heart-beat analysis and invasive ECG recordings, e.g., by placing an electrode on the fetal scalp during labor. Although this is currently the only way to reliably measure fECG waveform, it can be accomplished only under limited clinical circumstances, presenting a risk to the fetus' safety.

Fig. (1) illustrates an example of a short segment of fECG recorded both invasively (upper trace) through a fetal scalp electrode, and non-invasively (lower two traces) through electrodes placed on the mother's abdomen. Four fetal beats (labeled a, b, c and d) are circled on all three traces. Note the abdominal traces contain much smaller fetal beats embedded in significant broadband noise, and much larger amplitude artifacts (transient oscillations) which are due to the mother's heart ${ }^{1}$. Note also that the artifacts manifest both in between and on top of fetal heart beats, with a similar morphology to the fetal heart beats.

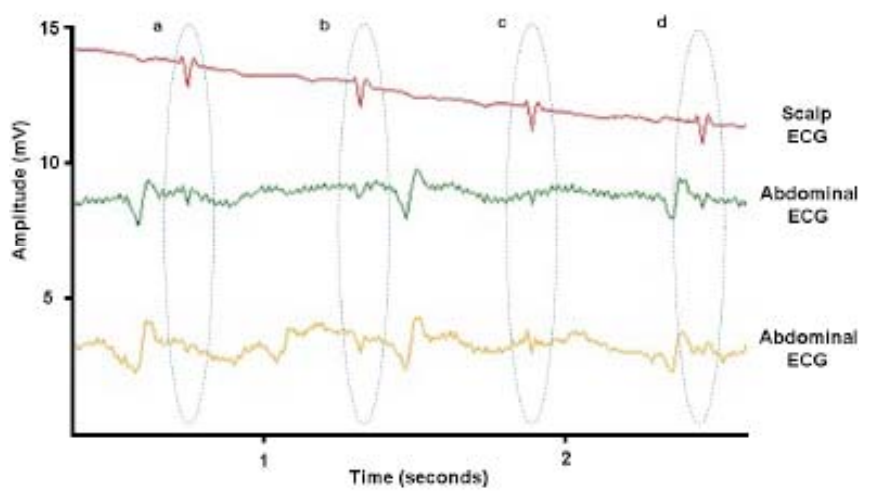

Fig. (1). Fetal ECG recorded invasively (upper trace) through a fetal scalp electrode, and non-invasively (lower two traces) through electrodes placed on the mother's abdomen. Fetal heart beats are circled and labeled $\mathbf{a}, \mathbf{b}, \mathbf{c}$ and $\mathbf{d}$.

In Fig. (2) the amplitude and frequency range of fECG have been compared with other biosignals and artifacts. Accordingly, the fECG is much weaker than the other biosignals. Moreover, from the signal processing perspective, there is no specific domain (time, space, frequency, or feature) in which the fECG can be totally separated from the interfering signals. Fig. (3) illustrates the fact that the main part of the fetal heart beat on the electrocardiogram (the QRS complex) lies in the same frequency domain as the adult QRS complex, as well as broadband muscle noise.

\section{HISTORICAL REVIEW OF EARLY WORK}

The fetal electrocardiogram was first observed by $\mathrm{M}$. Cremer in 1906 [13]. The early works in this area were performed by using the galvanometric apparatus of that time, which were limited by the very low amplitude of the fetal signals. As measurement and amplification techniques improved, fetal electrocardiography became more feasible and popular $[14,15]$. The limiting factor was then the low fetal SNR, especially in presence of the strong maternal

${ }^{1}$ Here we distinguish continuous noise from rapidly transient artifacts due to their different origins and the signal processing techniques adopted for their removal. cardiac interference; a problem which exists up to now. In the 1960's intra-uterine electrodes (placed between the intact membranes of the fetus and the wall of the uterus) provided SNR improvement for fECG analysis [16]. however, the technique was short lived due to the inherent danger of premature rupture of the membranes induced by the insertion of the electrode. Hon [17] introduced the use of the first direct electrode, inserted through the cervix, and fixed to the presenting part of the fetus after rupture of the membranes (typically to the fetal scalp).

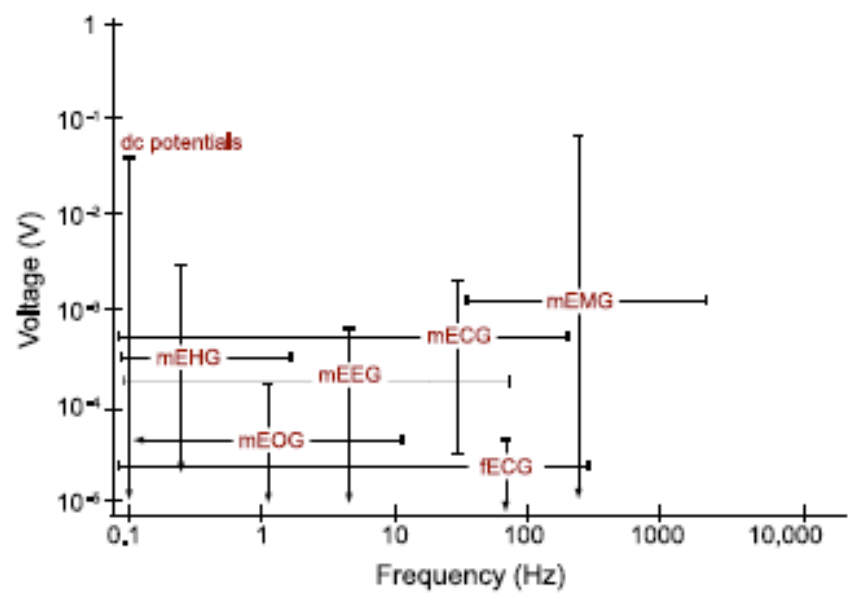

Fig. (2). The amplitude and frequency range of different biosignals, some of which interfere with fetal cardiac signals [9-11]. The labels in this figure stand for the maternal electrocardiogram (mECG), electroencephalogram (mEEG), electrooculogram (mEOG), electromyogram (mEMG), electrohystrogram (mEHG), and the fetal ECG (fECG). Note that the amplitude of these signals also depends on the site from which the data is recorded.

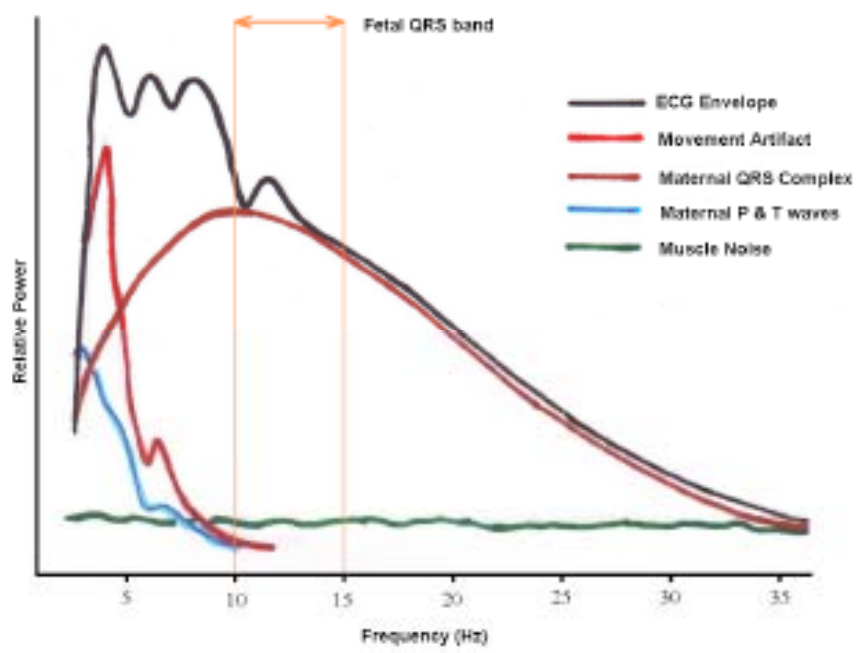

Fig. (3). A general representation of the signal, artifacts and noise present in the ECG in the frequency domain. The region in which the fECG QRS complex manifests is also marked. Note that the fECG largely overlaps with the maternal ECG and other interference. The fECG information also overlaps with the undesired signals in time, space, and feature domains as well; therefore, methods that only work in one specific domain are not able to fully separate the fetal cardiac signals. Image adapted from [12]. 
Shortly afterwards, with the developments in computer science and signal processing techniques, automatic signal processing and adaptive filtering techniques were used for fetal R-wave detection [18], and maternal cardiac interference cancellation from abdominal electrodes $[19,20]$. However, the techniques never provided more that approximate fetal heart rate estimates, and the issue of fECG analysis has since been considered as challenging problem for both the biomedical and signal processing communities.

In order to quantify the volume of past and current research interest in this field, the number of publications in the field of fetal electrocardiography and magnetocardiography, which have been registered in PubMed [21], can be seen in Fig. (4). We can see that after a sharp peak in the 1960 's, the trend seems to have been decreasing until the year 2000 (of course in terms of the number of publications). But in the last decade, the interest has again increased, especially for fetal magnetocardiography. This should be partially seen as a result of novel low-noise and low-price measurement and digitizing systems, and partially due to the developments in array signal processing and adaptive filtering techniques. Of course, comparing this with the total number of publications in the field of electrocardiography and magnetocardiography (both for adults and fetuses) in Fig. (5), we notice that fetal cardiography is still in its preliminary stages and there is still a long way to go, for making fetal cardiography a clinically trustable means of fetal cardiac monitoring. It should also be noted that as illustrated in Fig. (6), despite the increase in the number of investigations involving the electrcardiogram or magnetocardiogram (MCG: see section 4.2) in Fig. (5), when normalizing the number of these works by the total number of publications registered in PubMed over the same period, we notice that the percentage of studies in ECG studies has decreased since the 1980's, while MCG research has gained more interest. In section 5, we categorize some of the existing methods in this area from different viewpoints and describe their merits and drawbacks.

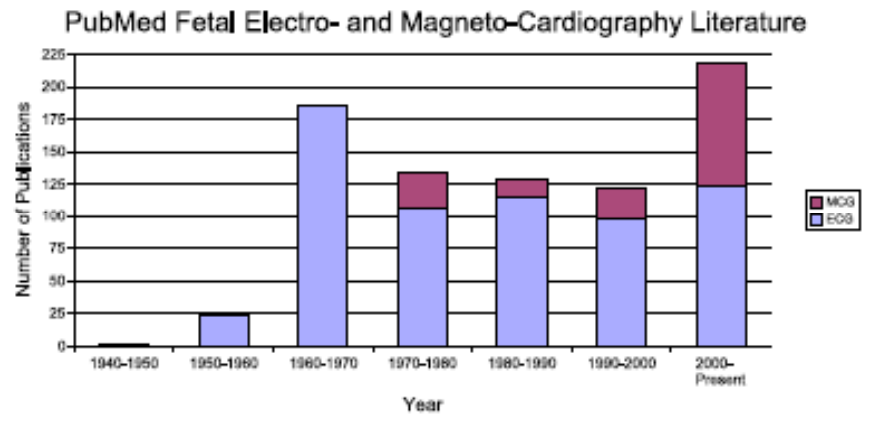

Fig. (4). Number of publications in the field of fetal electrocardiography and magnetocardiography, registered in PubMed [21].

\section{PHYSIOLOGY OF THE FETAL HEART}

\subsection{Fetal Heart Development}

The heart is among the first organs developed in the fetus and undergoes a considerable amount of growth in the very early stages of pregnancy [22] (Fig. 7). The most critical period of this development is between 3 and 7 weeks after fertilization, when a simple heart tube assumes the shape of a four-chambered heart (Fig. 8).

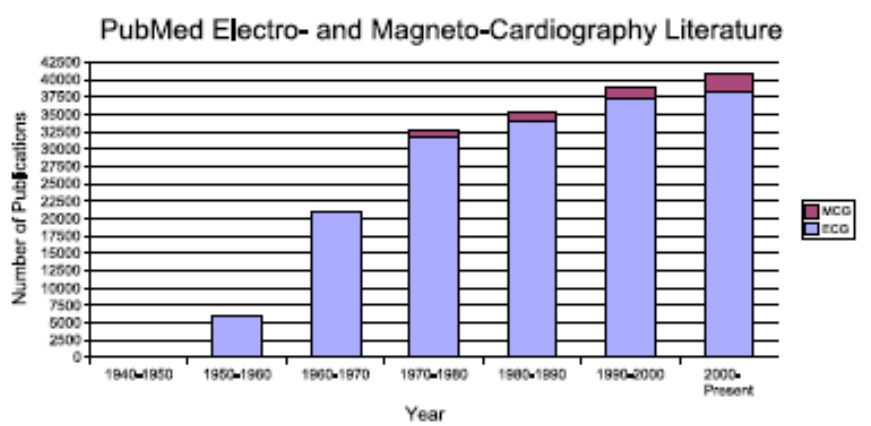

Fig. (5). Number of publications in the field of electrocardiography and magnetocardiography, registered in PubMed [21].

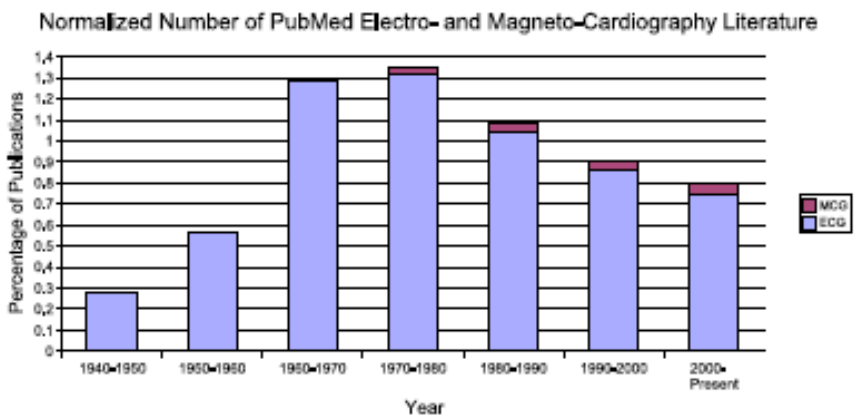

Fig. (6). The percentage of publications in the field of electrocardiography and magnetocardiography normalized by the number of publications in all subjects, registered in PubMed [21].

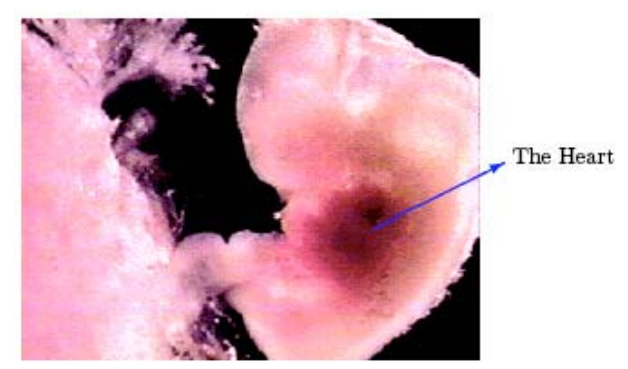

Fig. (7). The fetus and its heart in the early stages of development; adapted with permission from [24].

The heart is believed to begin beating by the 3 rd week of life and pumps its own blood through a separate closed circulatory system with its own blood type. Shortly after, the eye, ear and respiratory systems begin to form. The fetal heart can then be externally monitored by ultrasound imaging in the 7th to 9th week [22]; although only vague images are recordable at this step. The cardiac waveforms and beat-to-beat variability of the heart rate are not measurable in ultrasound imaging. At 20 weeks, the fetal heart beat can be heard without amplification, with a rate of around 120-160 beats per minute, but little extra diagnostic information can be ascertained from auscultation above heart rate.

Therefore, the fECG and MCG that contain morphological information of the cardiac activity have 


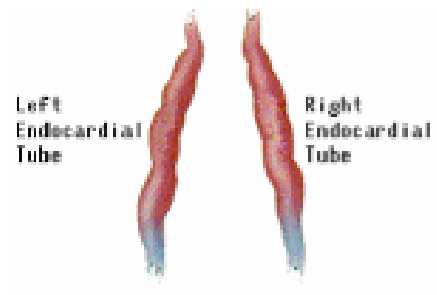

(a)

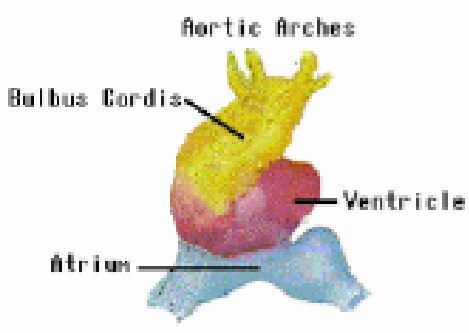

(d)

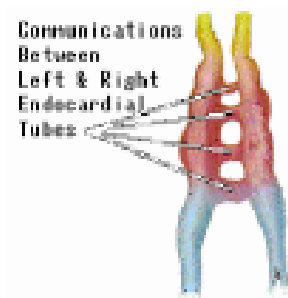

(b)

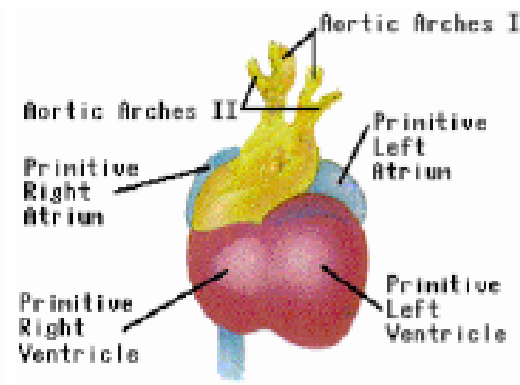

(e)

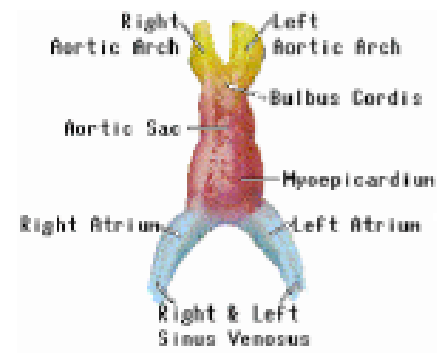

(c)

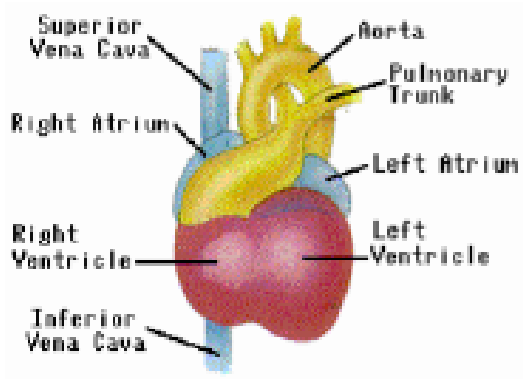

(f)

Fig. (8). Development stages of the fetal heart during gestation in chronological order (from a to f); adapted with permission from [24].

received much interest. These signals can be recorded from the maternal abdomen as early as the eighteenth to twentieth week after conception [8, 23].

At 6 weeks post fertilization, brain waves can be recorded, the skeleton is complete and most reflexes are present. However, the embryo is only about $0.5 \mathrm{~cm}$ in length and the head, mouth, liver, and intestines are just beginning to take shape. At 12-14 weeks (when the fetus weighs around $30 \mathrm{~g}$ and is about $7 \mathrm{~cm}$ long), the muscles begin to develop and spontaneous movements can be observed which intensify with age.

Rather late into the pregnancy, at around 26 weeks, the fetus begins to inhale and exhale. Of course, the fetus is not breathing air and oxygenating the blood through this action. This is thought to aid with the production of surfactant and exercise the respiratory system before birth. Although the fetus is capable of living outside the womb at 23-24 weeks, this would be considered premature at birth, and the normal gestation time is approximately 40 weeks [25]. In fact, at 23 weeks there is only a $15 \%$ chance of viability outside of womb with the viability probability rising to $56 \%$ at 24 weeks and $79 \%$ at 25 weeks.

\subsection{Fetomaternal Compartments}

A simplified anatomy of the fetomaternal compartments can be seen in Fig. (9). Accordingly, the fetus is surrounded by several different anatomical layers with different electrical conductivities [26, 27]. The highest and lowest conductivity are found in the amniotic fluid and the vernix caseosa, respectively. Both of these layers surround the fetus completely.

In the maternal abdomen compartments, the skin and the subcutaneous fat also have a poor conductivity -- about ten times smaller than the muscle tissue [27, 28]. Therefore these

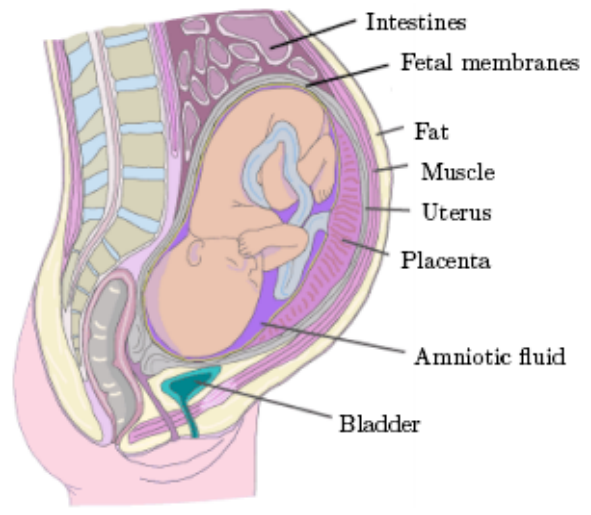

Fig. (9). The major fetomaternal compartments that influence the fetal cardiac surface potentials; adapted with permission from [28]. The vernix caseosa is formed over the fetal skin.

two layers, which are the interface of the surface electrodes and the internal tissues, have considerable influence on the recorded fECG. All of these different tissues and layers form a so called, volume conductor, in which the fetal cardiac signals propagates up-to the maternal body surface. This volume conductor is not a steady conductor and its electric conductivity and geometric shape constantly change throughout gestation. Specifically, in the second half of gestation (20th week onward), in which the ECG and MCG are recordable from external electrodes, it is known that the amniotic fluid [29, 30], the placenta, and the fetus itself [31] are all increasing in volume. The very low conductivity vernix caseosa layer is formed between the 28th and 32 nd weeks of gestation [26, 27]. It almost electrically shields the fetus and makes the recording of fetal surface ECG very difficult. However, for normal pregnancies (non-premature deliveries), the layer slowly dissolves in the 37 th to 38 th 
weeks of pregnancy [28]. In previous studies, different interpretations have been presented for the fECG signals recorded during the third trimester of gestation (after the forming of the vernix caseosa), based on preferred current pathways such as the umbilical cord, the oronasal cavity, or even random holes in the vernix caseosa [26, 27]. The preferred current pathway hypothesis, besides the intrinsic weakness of the fECG and its gradual development, also explains some of the differences between adult and fECG and vectorcardiogram shapes.

\subsection{Fetal Presentations}

During the first two trimesters of pregnancy the fetus does not have a specific presentation and moves about a lot. By the middle of the third trimester the fetus commonly settles in a head-down position known as the vertex presentation, which is more appropriate for birth [32]. However, as shown in Figs. (10 and 11) the fetus may also settle in other less probable presentations. The presentation of the fetus influences the fetal cardiac signals recorded from the maternal body surface over different leads [33].

\subsection{Anatomy of the Fetal Heart}

The anatomy of a fully developed fetal heart is depicted in Fig. (12). There are some functional differences between the fetal and adult hearts. It is known that after birth, the left ventricle pumps blood to the body and the right ventricle pumps the blood to the lungs for acquiring oxygen. However, for the fetus the fetal oxygen is supplied by the placenta; therefore the blood is no longer pumped to the lungs for this purpose. Instead both ventricles pump the blood throughout the body (including the lungs) [28]. For this purpose there are two shunts, namely the the foramen ovale and the ductus arteriosus that link the outgoing vessels of both ventricles. This allows blood to enter the right atrium and to bypass the pulmonary circulation. A similar adaptation in the fetus is the ductus venosus, which is a vessel that allows blood to bypass the liver. It carries blood with oxygen and nutrients from the umbilical cord straight to the right side of the fetal heart [34].

After birth, the foramen ovale closes with the first breaths and the ductus arteriosus partially closes in 10 to 15 hours after birth and takes up-to three weeks for complete closure. The ductus venosus also closes shortly after birth, when the umbilical cord is cut and blood flow between the mother and fetus stops [34]. There are also other minor changes in the physiology of the baby's heart and its circulatory system that take place within the first year after birth.

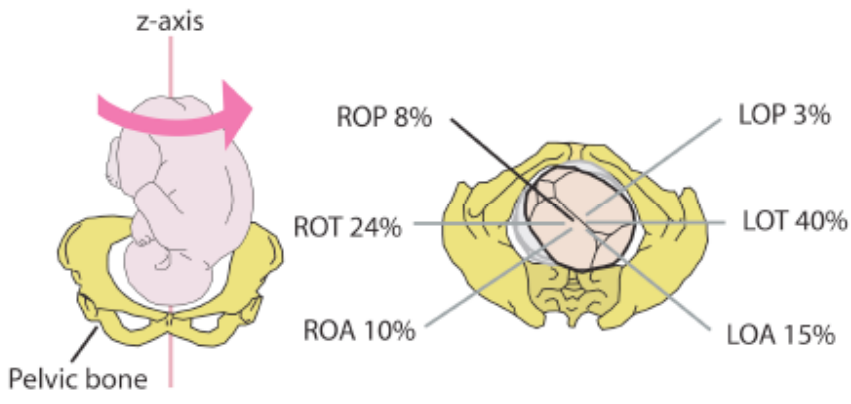

Fig. (11). Different fetal vertex positions and their incidence at the end of gestation; adapted with permission from [28]. In the right side abbreviations, $\mathrm{L}$ stands for left, $\mathrm{R}$ for right, $\mathrm{O}$ for occiput, $\mathrm{A}$ for anterior, $\mathrm{T}$ for transverse and $\mathrm{P}$ for posterior. The LOA position is seen in the left graph.

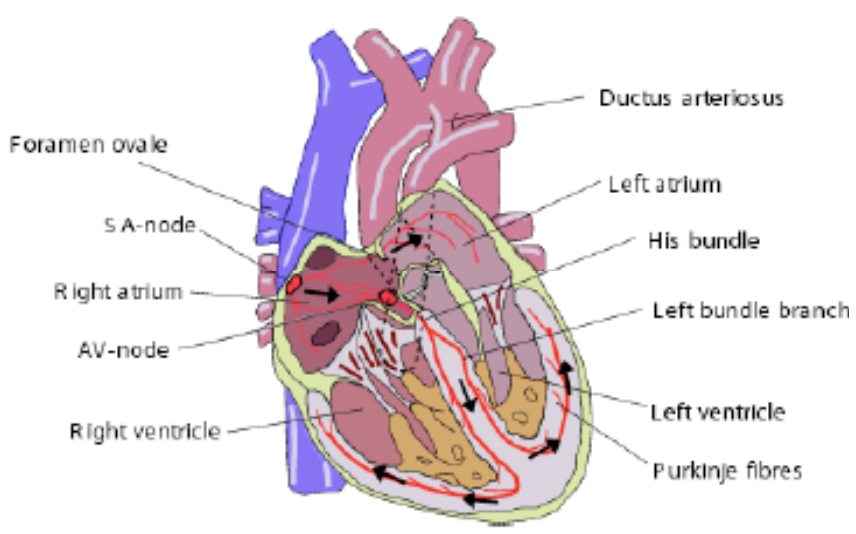

Fig. (12). The anatomy of the fetal heart; adapted with permission from [28].

\subsection{Electrical Activity of the Fetal Heart}

While the mechanical function of the fetal heart differs from an adult heart, its beat-to-beat electrical activity is rather similar. The wave-like pumping action of the heart is controlled by a network of neural fibers that are distributed throughout the myocardium and coordinate its regular contraction and relaxation [35]. The myocardial stimulation starts from the sinoatrial node (SA-node), which serves as the natural pacemaker for the heart. The SA-node is a cluster of cells located in the upper-right posterior wall of the right atrium, which sends the electrical impulse that triggers each heartbeat. This impulse further stimulates the second cluster of cells, namely the atrioventricular node (AV-node) that is situated in the lower posterior wall of the right atrium. After

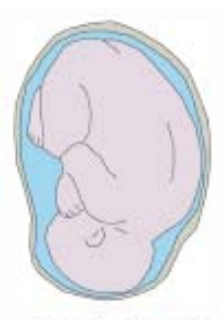

(a) Vertex $96.8 \%$

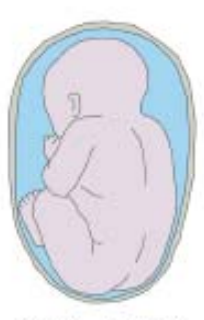

(b) Breech $2.5 \%$

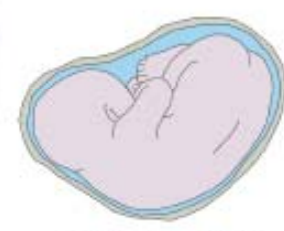

(c) Shoulder $0.4 \%$

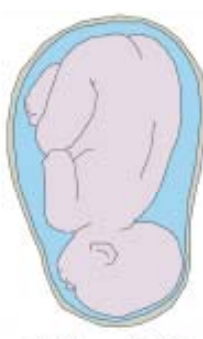

(d) Face $0.2 \%$

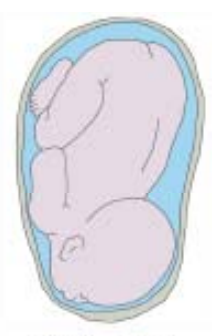

(e) Brow $0.1 \%$

Fig. (10). Different fetal presentations and the percentage of incidence at the end of gestation; adapted with permission from [28]. 
the AV-node, the depolarization front enters the bundle of His, the left and right bundles, and ends in the Purkinje fibers, depolarizing the ventricular muscles in its way.

The procedure of myocardium contraction is known as the depolarization (or systole) cycle that is followed by the repolarization (or diastole) cycle, in which the myocardium relaxes and becomes ready for the next activation. A complete cardiac cycle is depicted in Fig. (13).

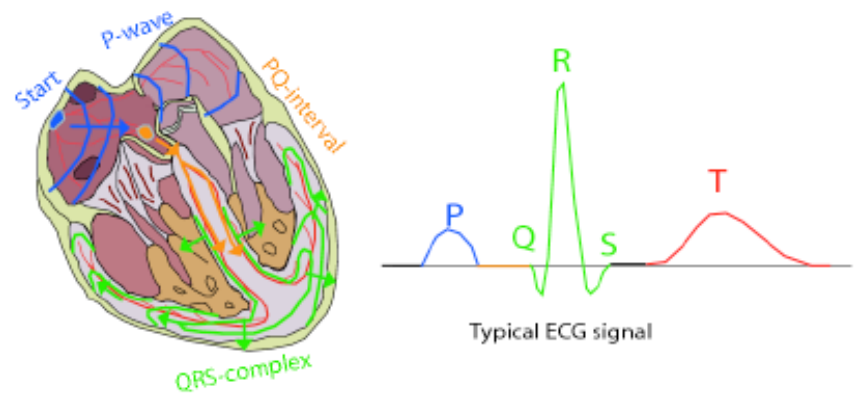

Fig. (13). The activation cycle of the fetal heart; adapted with permission from [28].

\subsection{The Electrocardiogram Morphology}

The ECG measured on the body surface is a result of the stage-wise activation of the myocardium and results in the so-called PQRST-complex depicted in Fig. (13). This letter representation was first coined by Einthoven in 1895 [36, 37]. In this notation, the P-wave accounts for the spreading of the depolarization front through the atria. During the next $50 \mathrm{~ms}$, only very weak signals are recordable, as it takes some time for the depolarization front to travel through the $\mathrm{AV}$-node and since only a small number of myocardiac cells take part in the atrioventricular conduction the signals are very small ${ }^{2}$ [38]. Next, the ventricles are depolarized resulting in the QRS-complex. At the same time the atria are repolarized; however this repolarization is obscured by the depolarization of the ventricles. Finally we have the T-wave, which corresponds to the repolarization of the ventricles. In some measurements the T-wave is followed by a small wave known as the U-wave, which for normal ECG, is believed to be due to repolarization of the His-Purkinje system [37]. Other hypotheses for the origin of the U-wave have been suggested in [39]. The segment between the end of the Uwave up-to the beginning of the next P-wave is known as the isoelectric segment of the ECG, in which the myocardium does not have a measurable activity.

Morphologically, adults and fetuses have rather similar ECG patterns; but the relative amplitudes of the fetal complexes undergo considerable changes throughout gestation and even after birth. The most considerable change concerns the T-waves, which are rather weak for fetuses and newborns [23].

\subsection{Fetal Heart Rate and Heart Rate Variability}

The changes in fetal heart rate (FHR) and fetal heart rate variability (FHRV) are not well understood, particularly

${ }^{2}$ Note that although frequently addressed as being isoelectric, the PQ segment is not isoelectric [38]. since the fetal central nervous system may not be fully formed at birth. Although there is no direct neural link to the fetus from the mother, it is thought that maternal activity can affect the fetal heart rate and blood pressure indirectly through the action of transmitted hormones (cortisol/ catecholamines) and possibly directly through compromised placental vasculature or blood volume [40-42]. Moreover, the maternal and fetal HR can become entrained, but the mechanism of entrainment is not clear [40]. In general though, the mother and fetus(es) have separate autonomic nervous sytems which are indirectly linked (mostly with the mother affecting the fetus) by changes in blood pressure and hormones.

At 20 weeks, the FHR has a baseline value of around 120 to 160 beats per minute (BPM). However, although the variations of the fetal heart rate are different to children and adults, the FHR is known to possess circadian variations with evolve over the course of the pregnancy [43]. Structurally, the heart-rate variability (HRV) of the fetus is also known to be simpler (less dynamic) than an adult. However, as the fetal autonomic nervous system evolves, the HRV patterns also become more and more complex $[44,45]$. A typical example of this complexity is depicted in Fig. (14).

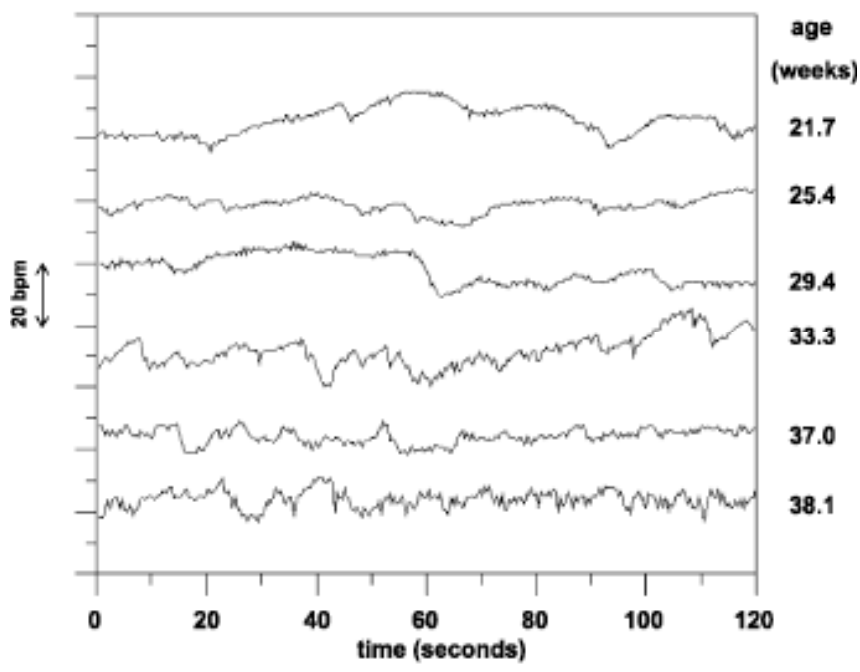

Fig. (14). The increasing complexity in fetal HRV time-series in different gestational ages; adapted with permission from [45].

Recently Muro et al. [46] made FHR recordings at 21, $24,27,30,33$, and 36 weeks from a normal pregnant woman and found significant diurnal variations in all the recorded gestational ages. Furthermore, they found the amplitude of diurnal variation increased with advancing gestation. The FHR baseline recorded at night (midnight-9:00 hours) significantly decreased from 24 to 30 weeks, while the afternoon/evening FHR baseline (15:00-21:00 hours) decreased after 27 weeks. These findings suggest that the changes in the diurnal variations of FHR baseline with advancing gestation reflect the process of development of the fetal nervous system.

Lunshof et al. [47] showed that a significant diurnal rhythm in basal FHR was present in 73\% of their fetal study population, and this rhythm was closely related to the maternal heart rate rhythm. They also found that diurnal 
rhythms in FHRV, accelerations, and activity were present in only $30 \%$ to $50 \%$ of the fetuses. Lange et al. found that FHRV changed with gestational age and mean HR, or activity levels, but not on gender or time of day.

It is also known that number of heart rate accelerations and decelerations per hour that a fetus experiences may also be related to its health, and is a function of gestational age. The rate and duration of episodes of sustained fetal tachycardia $(\mathrm{SFT})^{3}$, increases with gestational age [48]. Shono et al. [48] analyzed $24 \mathrm{~h}$ FHR in 102 normal pregnant women (at 30-41 weeks) to determine the gestational age related changes in SFT. For 38-39 weeks, nocturnal SFTs (19:00-07:00 hours) were significantly more frequent than during day-time (07:00--19:00 hours). At 30-31 weeks of gestation, there were no significant episodes, but in the 40th week, the rate and average duration increased to $85 \%$ and $114 \mathrm{~min}$.

\subsection{The Diagnostic Fetal ECG}

Currently fECG analysis is used in the clinical domain almost entirely to analyze heart rate and the associated variability. Morphological analysis of the fECG is generally not undertaken for cardiac-anomaly populations for the following reasons:

- Although structural heart defects are the most common structural fetal defect, they are rare (present in less than $1 \%$ of the population).

- Structural defects are evaluated using echocardiography, because the problem is structural, not conductive.

- There is no evidence that structural defects have abnormal conduction (although this has never been studied in-utero) or that it would be useful in clinical predictive medicine.

- By far the most common arrhythmia is premature atrial contractions, which are usually benign.

- Pathologic fetal heart block arrhythmias are extremely rare, and usually occur in patients with systemic lupus erythematosus (SLE).

One notable exception is the recent arrival of commercial monitoring systems that provide proxy ST-segment analyses using the waveform from the fetal scalp electrode (e.g. the STAN monitor from Neoventa Medical, Goteborg, Sweden). Beginning in the late 1990s, European investigators began using the STAN monitor in routine clinical practice. This system is based on an analysis of the quantitative ratio between the amplitude of the fetal T-wave and the fetal Rwave and on the presence or absence of a biphasic ST segment. The ratio is used clinically either to provide reassurance or raise concern in the event of a non-ominous but non-reassuring fetal heart tracing. The physiologic basis for this test is the observation that repolarization of the adult myocardium is extremely sensitive to hypoxia, resulting in elevation of the ST-segment in adults with coronary artery disease. Similar findings have been demonstrated in fetal sheep, with experimental hypoxia leading to elevation in the

\footnotetext{
${ }^{3}$ An increase in FHR from $160 \mathrm{BPM}$ or more and persisting for more than 1.5 to 2
} hours is considered to be SFT.
ST-segment and T-waves in the fetal ECG [49]. The change in this ratio is thought to represent altered cellular ionic currents during anaerobic cardiac metabolism [50, 51].

This metric that compares the voltage amplitude of two components of the ECG waveform (the T-wave and the Rwave) can be accomplished reliably even in the absence of a standard ECG vector. This characteristic is critical because the fetal position is uncertain and subject to change, and thus vector-based analyses are inherently problematic in a fetal population. A series of clinical trials were begun that compared use of the STAN algorithm in addition to standard fetal monitoring with fetal monitoring alone. Early results demonstrated that use of STAN helped to identify cases of intra-partum acidosis [52-56].

One of the inherent limitations of this approach to fetal cardiac monitoring, however, is that it requires placement of a fetal scalp electrode, which is possible only during labor. Moreover, only one dimension of the electrical activity of the heart can be imaged using this type of electrode. This means that the sensitivity of such a technique is particularly limited by noise and is confined to problems that affect the entire heart. Activity that affects other portions of the heart, or is only a subtle effect, will be missed by such electrodes. Another issue that affects the scalp electrode includes movement of the fetus, which will appear as axis movements. If several ECG leads were available, such problems could potentially be addressed, providing more specific analysis, earlier in pregnancy.

\section{DATA COLLECTION}

An essential component to evaluating any fECG analysis algorithm is a representative and high quality database. Fetal ECG data collection can either be invasive or noninvasive. In invasive methods, the recording electrodes have direct contact with the fetal skin, which may only be achieved by using an intra-uterine electrode during labor [57-59]. The signals recorded by invasive methods have better quality as compared with noninvasive methods; but the procedure is rather inconvenient and is limited to recordings during labor. On the other hand, noninvasive methods use the signals recorded from the maternal abdomen, which can be done in any stage of pregnancy using dozens of electrodes. However, the low fECG SNR and the other interferences are the limiting factors of this method. Nevertheless, due to the numerous advantages of the noninvasive method, a large body of research has been conducted towards the development of signal processing techniques for retrieving the fECG from noninvasive recordings.

\subsection{Modern Data Collection Techniques and Open Databases}

When assembling (or using) a fECG database it is essential to consider the number of electrodes, type of electrode, heterogeneity of the patient population, conditions and gestational ages, as well as the data quality (in terms of presence of noise, sampling frequency, and amplitude resolution). In terms of recording quality, for morphological analysis, one would expect that doubling or trebling adult requirements would be a suitable approach, leading to sampling frequencies of around $2 \mathrm{kHz}$ and resolution of 16 
bits. No current standards exist for electrode location, but concentric circles on the abdomen, covering all available angles will provide the maximal coverage.

Currently there are two public databases widely used by the signal processing community. The first, known as the Daisy dataset [60], consists of a single dataset of cutaneous potential recording of a pregnant woman. A total of 8 channels (4 abdominal and 3 thoracic) are available, sampled at $250 \mathrm{~Hz}$ and lasting 10 seconds. This dataset is part of a database know as the SISTA (Signals, Identification, System Theory and Automation) database from the SISTA group of the department of Electrical Engineering of the Katholieke Universiteit Leuven, Belgium.

The second database, known as the Non-Invasive Fetal Electrocardiogram Database, is a series of 55 multichannel abdominal fECG recordings taken from a single subject over a period of 20 weeks. this database was recently contributed to PhysioBank $[61]^{4}$. The recordings are in the EDF+ format, and include two thoracic (maternal ECG) signals and three or four abdominal (maternal+fetal ECG) signals. The signals were recorded at $1 \mathrm{kHz}, 16 \mathrm{bit}$ resolution with a bandpass filter $(0.01 \mathrm{~Hz}-100 \mathrm{~Hz})$ and a main notch filter $(50 \mathrm{~Hz})$. The recordings contain 2 thoracic signals and 3 or 4 abdominal signals. The data has been prepared for PhysioNet by the Digital Signal Processing Group (GPDS) of the Electronics Engineering Department, ETSE Escuela Técnica Superior de Ingeniería, University of València, Spain. However, no existing public database contains any associated clinical information or data from more than one subject. Moreover, there is no gold standard measurement in any of these databases (from the scalp electrode).

\subsection{Alternative Measurement Techniques}

In previous works, besides electrocardiography, the wellbeing of the fetal heart has also been monitored by other techniques [8], including:

- Echocardiography [62,63]; also known as sonography of the heart which is based on standard ultrasound techniques.

- Phonocardiography $[5,64,65]$; which is a graphic registration of the heart sounds and murmurs produced by the contracting heart (including its valves and associated great vessels), picked up as vibrations and transformed by a piezoelectric crystal microphone into a varying electrical output according to the pressure imposed by the sound waves $[66,67]$.

- Pulse Oximetry [68]; is used to measure oxygen saturation of fetal blood as an adjunct to electronic fetal heart monitoring. The amount of reflected infra-red light is used to determine the relative amount of oxygen in the blood under the fetus' skin. To do this, a custom catheter is passed through the dilated cervix, after the rupture of the membranes, to lodge against the cheek where the probe is sited. Fetal pulse oximetry is a relatively new fetal assessment technology. After consideration of the results of a multicenter randomized, controlled, clinical

${ }^{4}$ http://www.physionet.org/physiobank/database/nifecgdb/ trial on fetal pulse oximetry in the United States, the U.S. Food and Drug Administration approved the technology for clinical use on May 12, 2000. However, recent studies indicate that knowledge of the fetal oxygen saturation is not associated with a reduction in the rate of cesarean delivery or with improvement in the condition of the newborn [68], and the product is used only for research purposes.

- Cardiotocography [69]; which is the simultaneous measurement of the fetal heart rate with an ultrasound transducer, and the uterine contractions with a pressuresensitive transducer (called a tocodynamometer), for measuring the strength and frequency of uterine contractions [70].

- Magnetocardiography [28, 71-74] ; which is a technique to measure the magnetic fields of cardiac signals using extremely sensitive devices such as the Superconducting Quantum Interference Device (SQUID) [75].

Among these methods, echocardiography is perhaps the most commercially the most available means of fetal cardiac monitoring. However, the ECG and the MCG contain more information, since most cardiac abnormalities have some manifestation on the ECG or MCG morphology or RRinterval timing [8]. Note that due to the morphologic similarity of the MCG and ECG, MCG processing methods are similar to the ECG-based ones; although using the current SQUID technology for magnetic recordings, the SNR of the fetal MCG is usually higher than its ECG. However, ECG recording apparatus are simpler and currently more affordable as compared with MCG systems.

\section{DATA ANALYSIS}

We can categorize the existing fetal data analysis literature by their methodologies. The existing methods in this area include:

\subsection{Direct Fetal ECG Analysis}

In early works, fECG detection was done over raw data without any processing. For instance in [76], some special cases were reported in which due to the vertex presentation of the fetus, the fetal R-peaks appeared as positive peaks while the maternal peaks had negative peaks. In such cases, the detection of the fetal RR-intervals is rather simple and may be achieved by simple peak detection, even without the removal of the maternal ECG. However, these methods are not always applicable and highly depend on the fetal presentation and gestational age.

\subsection{Adaptive Filtering}

Different variants of adaptive filters have been used for maternal ECG cancellation and fECG extraction. These methods consist of training an adaptive or matched filter for either removing the maternal ECG using one or several maternal reference channels $[19,57]$, or directly training the filter for extracting the fetal QRS waves [18, 77]. Ad hoc, adaptive filters such as 'partition-based weighted sum filters' [78], and least square error fittings [79], have also been used for this purpose. 
Note that the existing adaptive filtering methods for maternal ECG artifact removal, either require a reference maternal ECG channel that is morphologically similar to the contaminating waveform, or require several linearly independent channels to roughly reconstruct any morphologic shape from the three references [19]. Both of these approaches are practically inconvenient and with limiting performance, since the morphology of the maternal ECG contaminants highly depends on the electrode locations and it is not always possible to reconstruct the complete maternal ECG morphology from a linear combination of the reference electrodes 5 . Therefore, a maternal ECG cancellation method that would not require any excess reference electrodes or at most a single reference without the morphologic similarity constraint is of great interest.

The Kalman Filtering framework, developed in [80-82], which can be considered as members of the general class of adaptive filters, are promising approaches that use only an arbitrary maternal ECG reference for maternal ECG cancellation and fECG enhancement. This framework can be extended to more advanced filter types such as the Particle Filter in future research.

\subsection{Linear Decomposition}

Decomposition of single or multi-channel recordings is another common approach. In this method, the signals are decomposed into different components by using suitable basis functions. The basis functions can be selected from classes that are somehow in coherence with the time, frequency, or scale characteristics of the fetal components. Wavelet decomposition [83, 84], and matching pursuits [85], are among these methods.

Spatial filtering techniques such as singular value decomposition (SVD) [20, 86-89], blind and semi-blind source separation [90], can be considered as data-driven decomposition methods, which construct the required basis functions from the data itself, by maximizing some statistical measure of signal separation. In [91, 92], it has been shown that for fECG extraction blind source separation methods outperform adaptive filters such as the one proposed in [19]. One of the advantages of spatial filtering over conventional adaptive filters is that they can also separate the maternal and fetal complexes with temporal overlap.

Different variants of blind and semi-blind source separation methods have been used for fECG extraction [93, 94]. These methods are commonly based on the assumption of independent components (or more generally independent subspaces) for the maternal and fetal signals, or the existence of some temporal structure for the desired signals [95, 96, 97]. Blind source separation methods have also been combined with wavelet decomposition for extracting and denoising the fECG signals [98, 99]. One of the key problems in blind source separation approaches is that there is an implicit assumption that the signals and noises are mixed in a linear and stationary manner. Since both the fetal and maternal signals move (as they breathe, and shift positions), the stationary mixing is often not a good approximation.

${ }^{5}$ See [80] for the limitations of the single dipole model of the heart.
Decomposition methods are currently the most common and effective way of fECG extraction and denoising. However, the existing methods are rather generic and have not been fully customized to the periodic structures of ECG. Therefore, a challenging issue is to design multichannel signal processing methods (linear or nonlinear) that are specific to ECG/MCG signals. One recently described approach [80, 100, 101] involving adaptive blind source decomposition techniques, combined with model-based signal processing has shown promising results. Figs. (15 and 16) illustrate typical results of these new approaches. Note in particular that the clinical features are preserved in these examples. Another notable feature of the extracted fetal ECG is that the respiratory influence on the QRS complex is preserved. This opens up the possibility that fetal respiration activity could be routinely monitored and investigations be performed to associate particular respiratory patterns with subsequent neonatal problems such as sudden infant death syndrome or neonatal apnea.

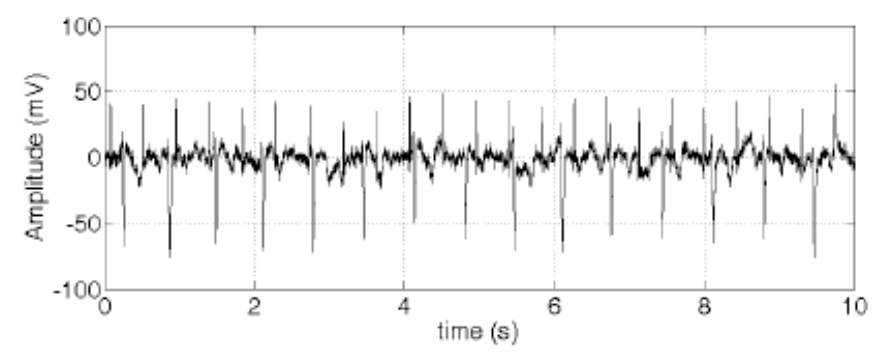

(a) Original

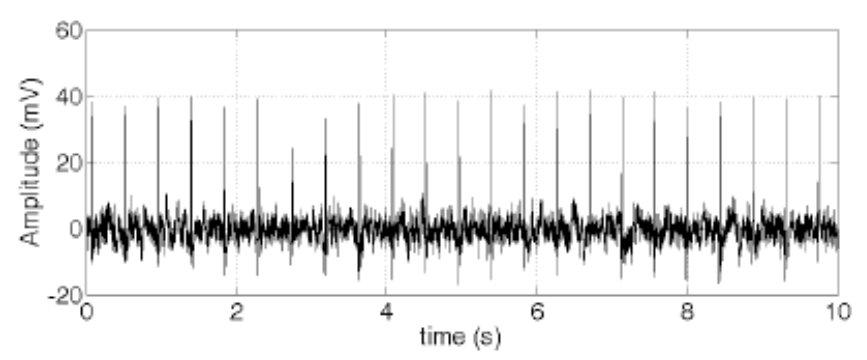

(b) After mECG removal

Fig. (15). Upper plot: Mixture of maternal and fetal ECG. Maternal beats are negative spikes $(\mathrm{HR}=90 \mathrm{BPM})$. Fetal beats are the smaller, positive spikes $(\mathrm{HR}=138 \mathrm{BPM})$. Lower plot: Extracted fECG (before denoising). Note that number of beats and relative positions are preserved and so fetal HR estimation and HRV is viable.

\subsection{Nonlinear Decomposition}

Linear decomposition methods using either fixed basis functions (e.g. wavelets), or data-driven basis functions (e.g. singular vectors) have limited performance for nonlinear or degenerate mixtures of signal and noise. In fact, fetal signals and other interferences and noises are not always 'linearly separable' [80]. One solution for such cases is to use nonlinear transforms for separating the signal and noise parts of the observations. Of course, nonlinear transforms are rather $a d$ hoc and require some prior information about the desired and undesired parts of the signal. In [102-105], using nonlinear projections, a series of maternal ECG cancellation and fECG enhancement methods have been developed. 
These methods consist of using the noisy signal and its delayed versions for constructing a state-space representation of the signal, smoothing the state-space trajectory using conventional or PCA smoothers [106], and transferring the samples back to the time-domain representation. These methods are very attractive from the point that they are applicable to as few as one single maternal abdominal channel. However, the selection of the required time-lags is empirical and the important inter-beat variations of the cardiac signals can be wiped-out during the state-space smoothing. Moreover, they have higher computational complexity as compared with linear methods, and the correct embedding dimension can changes as the noise statistics change.

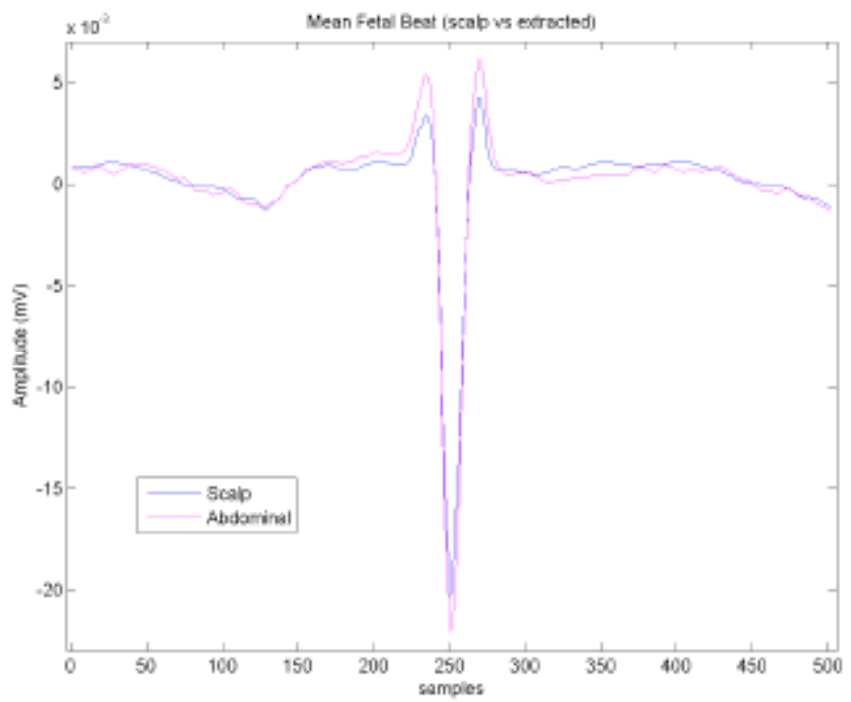

Fig. (16). Average fetal heart beat morphology taken from scalp electrode (blue line) and average fetal heart beat extracted from abdominal maternal/fetal mixture (magenta line). Note the preservation of clinical features (R-peak, QT-interval, ST-segment).

Another recently developed technique is a deflation method of subspace decomposition, which uses a combination of linear projection (and back-projections) with linear/nonlinear denoising schemes [101]. This method is very promising and has found various applications including the extraction of the fECG from low SNR channels with few number of electrodes [101].

In Table 1, a general comparison is made between the existing methods of fECG analysis. Accordingly, each method has its own benefits and limitations and is applicable for specific scenarios.

\subsection{Forward Modeling vs. Inverse Solutions}

An important aspect of noninvasive cardiac signal studies (either for adults or fetuses) is to find relationships between the cardiac potentials generated at the heart level and the potentials recorded on the body surface. This problem is known as the forward problem of electrocardiography, for which electromagnetic principles are used with electrophysiological models of cardiac potentials and volume conduction models, to predict the potentials that can appear on the body surface. Forward modeling also provides valuable insight for the more practical problem of estimating the cardiac potentials from body surface recordings, namely the inverse solution. Forward and inverse problems have long been studied for adult cardiac signals [107, 108]; but there are only few works of this sort for fetal cardiac signals.

Some pioneer studies on fetomaternal compartment conductivities and fECG modeling were presented in [26, 27, $109,110]$, where the fECG and the volume conductor medium from the fetal heart up-to the maternal abdominal leads were modeled and simulated. They have also questioned some previous results on the conductivity of the maternal internal body tissues and note that the fECG --as seen from the maternal abdomen-- does not have the same dimensionality throughout pregnancy. This may partially be due to the physiological changes of the fetal heart itself, and partially due to the low conductivity of the vernix caseosa that surrounds the fetus and electrically shields it from the outside world in certain gestational ages. Some of these findings have also been verified with experimental results.

In a more recent study, the problem of reliability of the fetal MCG and ECG has been studied using forward modeling in normal and pathologic cases [28]. They use different models for different stages of gestation. Specifically, in the forward modeling of the last trimester of gestation, they considered a layer of vernix caseosa containing two holes and achieved fECG maps that resembled the actual measured maps. The holes in the vernix caseosa were considered over the mouth of the fetus and the onset of the umbilical cord, corresponding to the preferred current pathways. Other issues concerning the low-amplitude of the fetal T-waves are also presented in this work. However, for the processing of their real data, they used simple methods such as average waveform subtraction of the maternal MCG.

\section{MODELING THE FETAL ECG}

\subsection{The Fetal Heart Source}

The myocardium, during its contraction, may electrically be considered as a surface of potentials. The resultant electrical activity of the heart has been modeled with various methods ranging from single dipole models to activation maps [111]. Among these methods, the simplest and yet the most popular is the single dipole model that is believed to explain $80 \%-90 \%$ of the representation power of the body surface potentials [112], and the ECG and VCG are based on it. According to the single dipole model, the cardiac electrical activity may be represented by a time-varying rotating vector, the origin of which is assumed to be at the center of the heart and its end sweeps a quasi-periodic region in the space.

This vector may be mathematically represented in the Cartesian coordinates, as follows:

$d(t)=x(t) \hat{a}_{x}+y(t) \hat{a}_{y}+z(t) \hat{a}_{z}$,

where $\hat{a}_{x}, \hat{a}_{y}$, and $\hat{a}_{z}$ are the unit vectors of the standard three vectorcardiogram body axes

With this definition, and by assuming the body volume conductor as a passive resistive medium which only attenuates the source field [107], any ECG signal recorded from the body surface would be a linear projection of the 
Table 1. Comparison of the Different Methods and their Applications

\begin{tabular}{|c|c|c|c|c|c|c|c|c|}
\hline & Method & $\begin{array}{l}\text { Overall } \\
\text { Performance }\end{array}$ & $\begin{array}{l}\text { SNR } \\
\text { Improvement }\end{array}$ & $\begin{array}{l}\text { Computa- } \\
\text { tional Cost }\end{array}$ & Realtime* & $\begin{array}{l}\text { Implementation } \\
\text { Complexity }\end{array}$ & $\begin{array}{l}\text { Operator } \\
\text { Interaction }\end{array}$ & Applications/Comments \\
\hline \multirow{6}{*}{ 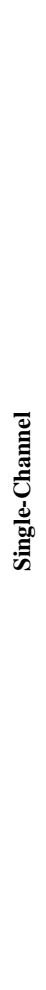 } & $\begin{array}{l}\text { FIR/IIR } \\
\text { Filtering }\end{array}$ & Low & Low & Low & Yes & Simple & Not required & Simple frequency domain denoising \\
\hline & $\begin{array}{l}\text { Wiener } \\
\text { Filtering }\end{array}$ & Medium & Medium & Medium & Possible & Medium & Not required & $\begin{array}{l}\text { Denoising using morphologic or } \\
\text { spectral priors; realtime implementa- } \\
\text { tion is only possible for the causal } \\
\text { Wiener filter }\end{array}$ \\
\hline & $\begin{array}{l}\text { Wavelet } \\
\text { Denoising }\end{array}$ & Medium & Medium & Low & Yes & Medium & Not required & $\begin{array}{l}\text { More robust denoising for } \\
\text { signal-noise mixtures having } \\
\text { different scales }\end{array}$ \\
\hline & $\begin{array}{l}\text { Adaptive } \\
\text { Filtering }\end{array}$ & Medium & Medium & Medium & Yes & Medium & $\begin{array}{l}\text { Sometimes } \\
\text { required }\end{array}$ & $\begin{array}{l}\text { Low quality maternal ECG cancella- } \\
\text { tion or fetal ECG enhancement } \\
\text { for fetal HRV analysis or average } \\
\text { morphologic studies; extendable } \\
\text { to multichannels }\end{array}$ \\
\hline & $\begin{array}{l}\text { Nonlinear } \\
\text { Filtering }\end{array}$ & Medium & Medium/High & High & No & Complex & Required & $\begin{array}{l}\text { Maternal ECG cancellation or } \\
\text { fetal ECG enhancement in single } \\
\text { channel recordings or degenerate } \\
\text { multichannel mixtures }\end{array}$ \\
\hline & $\begin{array}{l}\text { Kalman } \\
\text { Filtering }\end{array}$ & High & High & Median & Yes & Complex & $\begin{array}{l}\text { Sometimes } \\
\text { required }\end{array}$ & $\begin{array}{l}\text { ECG denoising, maternal ECG } \\
\text { removal or fetal ECG enhancement } \\
\text { having the R-peaks of the signal and } \\
\text { with rather consistent morphologic } \\
\text { shape; extendable to multichannels }\end{array}$ \\
\hline \multirow{4}{*}{ 语 } & $\begin{array}{l}\text { PCA/SVD/ } \\
\text { Factor } \\
\text { Analysis }\end{array}$ & Low & Low & Low & $\begin{array}{l}\text { Possible } \\
{[88]}\end{array}$ & Simple & Not required & $\begin{array}{l}\text { Dimension reduction } \\
\text { for high-dimensional } \\
\text { data and noise removal }\end{array}$ \\
\hline & ICA & Medium & Medium & Medium/High & No & $\begin{array}{l}\text { Medium/ } \\
\text { Complex }\end{array}$ & Required & $\begin{array}{l}\text { SNR improvement } \\
\text { not guaranteed }\end{array}$ \\
\hline & $\pi \mathrm{CA}$ & Medium & High & Low & No & Simple & Not required & $\begin{array}{l}\text { Decomposition of multichannel ECG } \\
\text { mixtures having the R-peaks of the } \\
\text { signal; dimension reduction with } \\
\text { minimal ECG information loss }\end{array}$ \\
\hline & $\begin{array}{l}\text { Subspace } \\
\text { Decomposition } \\
\text { by Deflation }\end{array}$ & High & High & Medium/High & No & Complex & $\begin{array}{l}\text { Sometimes } \\
\text { required }\end{array}$ & $\begin{array}{l}\text { Decomposition of (possibly) } \\
\text { degenerate multichannel mixtures } \\
\text { of desired and undesired subspaces } \\
\text { having a priori information about } \\
\text { the signal-noise structures }\end{array}$ \\
\hline
\end{tabular}

"Here we have not considered the possibility of block delayed realtime analysis.

${ }^{* * *}$ The indicated performances and complexities of multichannel methods are generally higher than single-channel methods; the qualitative descriptions should only be compared within each group separately.

dipole vector $d(t)$, onto the direction of the recording electrode axes $v=a \hat{\mathrm{a}}_{x}+b \hat{\mathrm{a}}_{y}+c \hat{\mathrm{a}}_{z}$ :

$E C G(t)=\langle d(t), v>=a \cdot x(t)+b \cdot y(t)+c \cdot z(t)$

In its most simplified form we can consider the dipole source of $d(t)$ inside a homogeneous infinite volume conductor. The potential generated by this dipole at a distance of $|r|$ is:

$\varphi(t)-\varphi_{0}=\frac{\mathbf{d}(t) \cdot \mathbf{r}}{4 \pi \sigma|\mathbf{r}|^{3}}=\frac{1}{4 \pi \sigma}\left[x(t) \frac{r_{x}}{|\mathbf{r}|^{3}}+y(t) \frac{r_{y}}{|\mathbf{r}|^{3}}+z(t) \frac{r_{z}}{|\mathbf{r}|^{3}}\right]$ where $\varphi_{0}$ is the reference potential, $\mathbf{r}=r_{x} \hat{\mathrm{a}}_{x}+r_{y} \hat{\mathrm{a}}_{y}+r_{z} \hat{\mathrm{a}}_{z}$ is the vector which connects the center of the dipole to the observation point, and $\sigma$ is the conductivity of the volume conductor [107, 113].

The ECG signals recorded from the body surface are the potential differences between two different points. Equation ((2)) therefore indicates how the coefficients $a, b$, and $c$ in ((1)) can be related to the radial distance of the electrodes and the volume conductor material. (In reality the volume 
conductor is neither homogeneous nor infinite, leading to a much more complex relationship between the dipole source and the body surface potentials.)

A 3-dimensional orthogonal vector representation of the ECG, (the VCG), is also possible by using three of the above models. Basically any set of three linearly independent ECG electrode leads can be used to construct the VCG. However, in order to achieve an orthonormal representation that best resembles the dipole vector $d(t)$, a set of three orthogonal leads that correspond with the three body axes are selected. The normality of the representation is further achieved by attenuating the different leads with a priori knowledge of the body volume conductor, to compensate for the non-homogeneity of the body thorax [113]. The Frank lead system [114], or the corrected Frank lead system [115] which has better orthogonality and normalization, are conventional methods for recording the VCG.

More generally, the cardiac signals may be represented by a multi-pole with $\mathrm{N}$ degrees of freedom. In [26] it has been discussed that the electrical activity of the fetal cardium is rather similar to an adult heart (especially in the second half of gestation), however due to the considerable distance between the recording electrodes and the fetal heart(s), and also due to the very low conductivity layers such as the vernix caseosa that (almost) electrically insulate the fetus from the outside world, the fetal electrical activity may be effectively modeled with a single dipole.

\subsection{The Propagation Media}

As suggested by Geselowitz $[107,116]$ since the effective frequency range of the ECG is below $1-2 \mathrm{KHz}$ and considering the distance between the body surface electrodes and the cardiac sources, the propagation medium of the maternal body may be considered as a linear instantaneous medium. The body surface recordings are hence a linear instantaneous projection of the cardiac sources and artifacts onto the axes of the recording electrode pairs. It is however known that the electrical impedance of the body volume conductor changes with respiration. Therefore despite of its linearity, the propagation medium is time-varying and the body surface recordings are rather nonstationary.

Seminal work performed by Oostendorp et al. [26, 27, $109,110]$ in the 1980's provides one of the most complete pictures of how the abdominal fECG transpires at the surface electrodes. In their work, they considered all the conductive media through which the fECG passes before being recorded at the mother's abdominal skin surface. Fig. (17) illustrates the media through which the current generating the fECG is conducted to the surface of the maternal abdomen (at approximately 30 weeks into gestation), where it is then recorded. Note that there are many layers of biomaterial, with differing dielectric constants. However, the largest difference between any of these layers is the vernix caseosa (which forms between weeks 22-30) which has a conductivity of $\sigma=10^{-7}$ to $10^{-5} \Omega^{-1} \mathrm{~m}^{-1}$. All other materials involved have a conductivity of $\sigma=10^{-2}$ to $10^{1} \Omega^{-1} m^{-1}$. Moreover, the vernix is the only material which introduces a significant frequency dependency of the conductivity or phase. In fact, the vernix can cause a phase shift up to $\pi / 2$ radians [109].

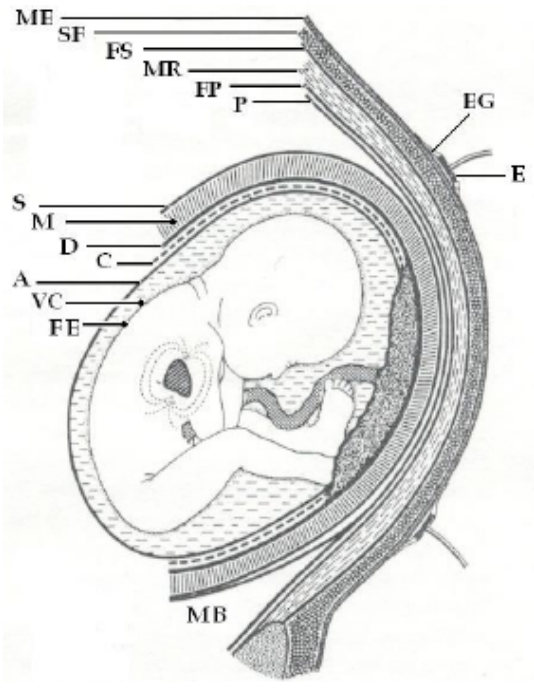

Fig. (17). Schematic representation of the media through which the current generating the fECG is conducted to the surface of the maternal abdomen at approximately 30 weeks. ME: Maternal Epidermis; SF: Subcutaneous Fat; FS: Fascia Superficialis; MR: Musculus Rectus Abdominalis; FP: Fascia Profunda; P: Peritoneum; E: Electrode; EG: Electrode Gel: S: Serosa; M; Myometrium; D: Decidua; C: Chorion; A: Amnion; VC: Vernix Caseosa; FE: Fetal Epidermis; MB; Maternal Bladder. Adapted from [26].

\subsection{A Synthetic Fetal ECG Model}

Based on the single channel ECG model proposed in [117] (and later updated in [118, 119], and [120]), the following dynamic model was suggested by Sameni et al. [121] for the $d(t)$ dipole vector:

$$
\begin{aligned}
& \dot{\theta}=\omega \\
& \dot{x}=-\sum_{i} \frac{\alpha_{i}^{x} \omega}{\left(b_{i}^{x}\right)^{2}} \Delta \theta_{i}^{x} \exp \left[-\frac{\left(\Delta \theta_{i}^{x}\right)^{2}}{2\left(b_{i}^{x}\right)^{2}}\right] \\
& \dot{y}=-\sum_{i} \frac{\alpha_{i}^{y} \omega}{\left(b_{i}^{y}\right)^{2}} \Delta \theta_{i}^{y} \exp \left[-\frac{\left(\Delta \theta_{i}^{y}\right)^{2}}{2\left(b_{i}^{y}\right)^{2}}\right] \\
& \dot{z}=-\sum_{i} \frac{\alpha_{i}^{z} \omega}{\left(b_{i}^{z}\right)^{2}} \Delta \theta_{i}^{z} \exp \left[-\frac{\left(\Delta \theta_{i}^{z}\right)^{2}}{2\left(b_{i}^{z}\right)^{2}}\right]
\end{aligned}
$$

where $\Delta \theta_{i}^{x}=\left(\theta-\theta_{i}^{x}\right) \bmod (2 \pi), \Delta \theta_{i}^{y}=\left(\theta-\theta_{i}^{y}\right) \bmod (2 \pi)$, $\Delta \theta_{i}^{z}=\left(\theta-\theta_{i}^{z}\right) \bmod (2 \pi)$, and $\omega=2 \pi f$, where $f$ is the beatto-beat heart rate.

The first equation in (3) therefore generates a circular trajectory rotating with the frequency of the heart rate. Each of the three coordinates of the dipole vector $d(t)$, is modeled by a summation of Gaussian functions with the amplitudes of $\alpha_{i}^{x}, \alpha_{i}^{y}$, and $\alpha_{i}^{z}$; widths of $b_{i}^{x}, b_{i}^{y}$, and $b_{i}^{z}$; and located at the rotational angles of $\theta_{i}^{x}, \theta_{i}^{y}$, and $\theta_{i}^{z}$. The intuition behind this set of equations is, that the baseline of each of the dipole coordinates is pushed up and down, as the trajectory approaches the centers of the Gaussian functions, generating a moving and variable length vector in the $(x, y, z)$ space. Moreover, by adding perturbations or deviations to the parameters of (3) (i.e. considering them as random variables rather than deterministic constants), it is possible to generate more realistic cardiac dipoles with inter-beat variations. 
Such a model, is of course, a simple approximation. However, it does provide a possible framework for testing signal processing algorithms. Various mixing models can be used to generate arbitrary projections onto a surface such as the maternal abdomen. See Sameni et al. [121] for more information.

\section{CURRENT CHALLENGES}

Despite of the richness of the literature, there are still several key areas that require further study in the field of fetal electrocardiography, particularly in the domain of multichannel noninvasive maternal abdominal measurements. The objective is to retrieve the fECG morphology with the highest possible fidelity, as required for morphological studies. In this context, the limiting factors and challenging signal processing issues include:

- weakness of fetal cardiac potentials and low conductivity layers surrounding the fetus which lead to low amplitude fECG at the maternal body surface;

- high interference of maternal ECG, uterus contraction, maternal respiratory, and motion artifact signals;

- possible movements of the fetus and the need for a sort of 'canonical representation' of the fetal cardiac signals with respect to the fetal body axis;

- development of automatic procedures that can be applied to long datasets with minimal interaction with an expert operator;

- providing confidence measures for the estimated cardiac signals and finding theoretical bounds for the amount of 'retrievable information' from body surface recordings in presence of noise.

Moreover, conventional ECG filtering methods are commonly based on some measure of time, frequency, or scale separability of signals and noise, which is common with all denoising techniques. However, cardiac signals have additional pseudo periodic structure, which appear not to have been well-utilized in ECG denoising schemes.

In previous studies multichannel decomposition methods have been commonly applied to the observed signals rather 'blindly' and there is generally no guarantee that the fetal components be extracted as separate components. Therefore, an important issue is to increase the probability of extracting the fetal components and also to improve the quality of the extracted components, through appropriate preprocessing and using a priori information about the signal/noise mixtures. This is a necessary step for developing robust fetal ECG/MCG extraction algorithms.

Linear decomposition methods are very common, not only due to the validity of linear model itself but also for the simplicity of these models. (A discussion about the validity of linear models for ECG decomposition is presented in [80].) However, as mentioned before, there are cases in which the desired signals are not linearly separable and nonlinear decomposition is inevitable. (See [80] for related discussions.) Therefore, an interesting field of study is to combine linear and nonlinear techniques to benefit from the simplicity of linear transforms and the power of nonlinear methods at the same time.

Another related issue is to find physiological interpretations for components extracted by multichannel source separation techniques. While these methods are commonly based on the maximization of rather abstract statistical criteria, such as statistical independence, it is not very evident what the resultant components physically correspond to, when applied to real data. For cardiac signals, this issue is more crucial, when we consider that the heart is a distributed source and not a point source.

Morphologic modeling of the fECG is another issue of interest. While previous fetal ECG/MCG models concentrate on forward models based on electromagnetic and volume conduction theories $[26,28]$, for evaluating signal processing techniques based on body surface potentials, more abstract models are required. In fact, in order to evaluate and compare single or multichannel processing techniques, we need models that enable us to manipulate the signal processing aspects of the simulated signals such as their morphology, RR interval timing, fetal position, dimensionality, and SNR, without going into the details of signal propagation and volume conduction theories. For adult ECG, an example of such models was developed in [117], where single channel adult ECG was modeled with a dynamic model. However, the existing models have not considered the multidimensional nature of the ECG and are not appropriate for the evaluation of multichannel techniques that use the 'mutual information' of the different channels.

\section{RECOMMENDATIONS AND FUTURE POSSIBI- LITIES}

These observations lead us to make several suggestions for future directions. First, a set of public gold standard databases are required, such as those for adult ECGs found at PhysioNet [61]. Such a database would need to contain high quality ECG (2 kHz, 16 bit) multichannel recordings of hundreds of normal and abnormal fetuses for several stages of pregnancy and different conditions. Accompanying clinical information such as outcome Apgar score, maternal medical state and history, and possibly limited genetic information should also be recorded, together with the results of tests such as amniocentesis. Clinical annotations should also be recorded, such as fetal location, contractions, movements, artifacts and clinical events (such as decelerations).

Since the exact (or even approximate) optimal combination of electrode positions is currently unknown, and likely to be dependent on the location and activity of the fetus, a large number of surface electrodes needs to be used (perhaps around 32), covering most of the abdomen. From this, it may be possible to determine (dynamically), which subset of electrodes provides the maximal amount of information for a given presentation [122]. Nevertheless, one should note that the registration of large number of robust signals from the abdomen in long recording sessions, is challenging from, both, the measurement and clinical aspect. The measurement aspect concerns the design of low-noise multichannel recording systems and the sensor development technology. The clinical aspect concerns the difficulty of attaching/detaching large numbers of sensors to/from the 
abdomen of pregnant women. In order to have good contact with the skin, the clinician has to clean the skin and firmly attach the sensors. This procedure is rather time-taking and stressful for the pregnant woman (even for volunteer subjects).

For dilated mothers, fetal scalp electrodes may have been placed and could provide a gold (or rather silver) standard by which we can compare the processed abdominal fECG. Comparisons of clinical parameters, such as ST-level, QTinterval and R-peak location (for HRV studies) should be compared to ensure that the extraction methods do not lead to clinically significant changes in these parameters (for both healthy and unhealthy subjects).

Such a database will allow the objective comparison of different signal processing techniques for fECG extraction. Without such a resource, we are unable to judge which algorithms provide the best performance for a given condition. Since complex real time processing is now possible on modern embedded systems, it is important that we assess how intelligent systems, neuro-fuzzy networks, morphological operators and other modern classification techniques can be employed to make the extracted information more useful for clinical applications.

One other area in which work is still required is that of modeling the fetal dipole and conductive medium of the womb, particularly in terms of personalized medicine. If patient-specific models can be made through simple noninvasive tests such as ultrasound- or impedance- based methods, then signal processing methods can use such information to make more accurate attempts at cleaning up the signal and extracting useful information.

Since robust and accurate fetal morphology extraction systems have not been properly evaluated, little work has been performed on the meaning of arrhythmias and changes in variability in the fetus. The underlying rhythms of the fECG, and associated phenomena such as respiration, require detailed investigations to determine what abnormal changes are indicative of adverse events, and can be used to provide decision support.

Finally, data fusion and information integration is almost a completely unexplored area of fetal monitoring. For better predictive performance and more accurate decision support, it is likely that a combination of cardiac activity recording modalities, such as the phonocardiogram, MCG, ultrasound, etc together with the invasive and/or non-invasive ECG will provide improved performance. Of course, simply adding data sources together does not guarantee more information, since extremely noisy signals may in fact lead to a reduction in the quality of the data. Therefore, the development of accurate and reliable signal quality metrics is essential. Signal quality metrics can be used either as a primary rejection scheme (whereby the channel is suppressed when the quality is below some pre-defined threshold) or can be used in a continuous fashion to unweight the contributions of a channel that is less trustworthy. For example, Li et al. [123, 124] have described a framework in which signal quality can be used to automatically used to reject variables derived from unreliable channels of data.
In conclusion, there are many areas in the field of fECG analysis which require attention before we can trust the results being produced by research algorithms and commercial devices. However, recent developments in signal processing are encouraging, and new developments in the field have the potential to positively impact patient care at little extra cost in terms of hardware.

\section{ACKNOWLEDGMENTS}

The authors would like to thank the anonymous reviewers and Dr. A. Wolfberg for their helpful comments in preparing this manuscript. Parts of this work are derived from Dr. Sameni's Ph.D. thesis and therefore we would like to thank Profs. C. Jutten and M.B. Shamsollahi (who cosupervised Dr. Sameni), Prof. A. Van Oosterom and Dr. T. Oostendorp for their cooperation in sourcing their early works, and Dr. J. Stinstra, Dr. C.D. Lawrence, and Prof. R. T. Wakai for permitting the usage of some of the figures of this article. We would also like to thank Dr. Wolfberg and Courtenay Pettigrew for their efforts in collecting some of the data used in this article, and Jay Ward and Jim Robertson of MindChild Limited for their work on the data collection system and data review.

\section{DISCLOSURES}

Both authors hold shares in MindChild Medical Inc., North Andover, MA, USA and patents in the field of ECG analysis.

\section{REFERENCES}

[1] Congenital Heart Defects; 2005. Available from: http: //www. marchofdimes.com/professionals/14332_1212.asp

[2] Minino AM, Heron MP, Murphy SL, Kochanek KD. Deaths: Final Data for 2004. National Vital Statistics Reports 2007; 55(19).

[3] Congenital Heart Defects in Children Fact Sheet; 2008. Available from: http: //www.americanheart.org/children

[4] Pajkrt E, Weisz B, Firth HV, Chitty LS. Fetal cardiac anomalies and genetic syndromes. Prenat Diagn 2004; 24(13): 1104-15.

[5] Zuckerwar AJ, Pretlow RA, Stoughton JW, Baker DA. Development of a piezopolymer pressure sensor for a portable fetal heart rate monitor. IEEE Trans Biomed Eng 1993; 40(9): 963-9.

[6] Vintzileos AM, Nochimson DJ, Guzman ER, Knuppel RA, Lake M, Schifrin BS. Intrapartum electronic fetal heart rate monitoring versus intermittent auscultation: a meta-analysis. Obstet Gynecol 1995; 149-55.

[7] Practice ACOG Bulletin. Intrapartum Fetal Heart Rate Monitoring: nomenclature, interpretation, and general management principles. USA: American College of Obstetricians and Gynecologists 2009; p. 109.

[8] Peters M, Crowe J, Piéri JF, et al. Monitoring the fetal heart non-invasively: a review of methods. J Perinat Med 2001; 29: 408-16.

[9] Webster JG, Ed. Medical instrumentation: application and design. 3rd ed. USA: John Wiley \& Sons 1998.

[10] Devedeux D, Marque C, Mansour S, Germain G, Duchêne J. Uterine electromyography: a critical review. Am J Obstet Gynecol 1993; 169(6): 1636-53.

[11] Snowden S, Simpson NA, Walker JJ. A digital system for recording the electrical activity of the uterus. Physiol Meas 2001; 22(4): 673-9.

[12] Tompkins WJ, Ed. Biomedical digital signal processing: C language examples and laboratory experiments for the IBM PC. Englewood Cliffs, NJ: Prentice Hall 1993.

[13] Cremer M. Über die Direkte Ableitung der Aktionstrome des Menschlichen Herzens vom Oesophagus und Über das Elektrokardiogramm des Fetus. Münchener Medizinische Wochenschrift 1906; 53: 811-3. 
[14] Goodyer AVN, Geiger AJ, Monroe WM. Clinical fetal elecrocardiography. Yale J Biol Med 1942; (15): 1-19.

[15] Lindsley DB. Heart and Brain Potentials of Human Fetuses in Utero. Am J Psychol 1942; 55(3): 412-6.

[16] Sureau C. Electrocardiographie foetale humaine normale. Bull Soc R Belg Gynecol Obstet 1960; 123(30).

[17] Hon EH. The instrumentation of fetal heart rate and fetal electrocardiography I. A fetal heart rate monitor. Conn Med 1960; 24 : 289-93.

[18] Farvet AG. Computer Matched Filter Location of Fetal R-Waves. Med Biol Eng 1968; 6(5): 467-75.

[19] Widrow B, Glover J, McCool JM, et al. Adaptive noise cancelling: principles and applications. Proc IEEE 1975; 63(12): 1692-716.

[20] van Oosterom A. Spatial filtering of the fetal electrocardiogram. J Perinat Med 1986; 14(6): 411-9.

[21] PubMed; 2008. Available from: http: //www.pubmed.gov/

[22] Jana L. Early Fetal Heart Development: 0-9 Weeks; 2004. Available from: http: //www.drspock.com/article/0,1510,5287,00.html

[23] van Leeuwen P, Lange S, Klein A, Geue D, Grünemeyer DH. Dependency of magnetocardiographically determined fetal cardiac time intervals on gestational age, gender and postnatal biometrics in healthy pregnancies. BMC Pregnancy Childbirth 2004; 4(6): 22

[24] Lawrence CD. Fetal Heart Development 1995. Available from: http: //user.gru.net/clawrence/vccl/chpt1/embryo.htm

[25] Allen MC, Donohue PK, Dusman AE. The Limit of Viability Neonatal Outcome of Infants Born at 22 to 25 Weeks' Gestation. Bull Soc R Belg Gynecol Obstet 1993; 329(22): 1597-601.

[26] Oostendorp T. Modeling the Fetal ECG. Ph.D. dissertation, K. U. Nijmegen, The Netherlands 1989.

[27] Oostendorp TF, van Oosterom A, Jongsma HW. Electrical properties of tissues involved in the conduction of fetal ECG. Biomed Eng Comput 1989; 27: 322-4.

[28] Stinstra JG. Reliability of the fetal magnetocardiogram. University of Twente, Enschede, The Netherlands 2001. Available from: http: // doc.utwente.n1/35964/

[29] Brace RA, Wolf EJ. Normal amniotic fluid volume changes throughout pregnancy. Am J Obstet Gynecol 1989; 161(2): 382-8.

[30] Magann EF, Bass JD, Chauhan SP, Young RA, Whitworth NS, Morrison JC. Amniotic fluid volume in normal singleton pregnancies. Obstet Gynecol 1997; 90(4 Pt 1): 524-8.

[31] Baker PN, Johnson IR, Gowland PA, et al. Measurement of fetal liver, brain and placental volumes with echo-planar magnetic resonance imaging. Br J Obstet Gynaecol 1995; 102(1): 35-9.

[32] Osei EK, Faulkner K. Fetal position and size data for dose estimation. Br J Radiol 1999; 72(856): 363-70.

[33] Roche JB, Hon EH. The fetal electrocardiogram. V. Comparison of lead systems. Am J Obstet Gynecol 1965; 92: 1149-59.

[34] Sexton MJ, Latson LA. Fetal blood flow 2005. Available from: http: //www.memorialhermann.org/

[35] Michael GF, Philip AR, Frederick J, et al. Physiology; 2006. Available from: http: //www.cardioconsult.com/Physiology/

[36] Einthoven W. Über die Form des menschlichen electrocardiogramms. Arch Gesamte Physiol 1895; 60: 101-23.

[37] Hurst JW. Naming of the waves in the ECG, with a brief account of their genesis. Circulation 1998; 98(18): 1937-42.

[38] Ihara Z, van Oosterom A, Hoekema R. Atrial repolarization as observable during the PQ interval. J Electrocardiol 2006; 39: 2907.

[39] Barr RC. Genesis of the Electrocardiogram. In: Macfarlane PW, Lawrie TTV, ed. Comprehensive Electrocardiology. Oxford: Pergamon Press 1989; p. 129-51.

[40] Horimoto N, Morokuma S, Nakano H. Does maternal blood cortisol entrain fetal diurnal rhythm? Early Hum Dev 2004; 76(1): 5564.

[41] Maggioni C, Cornelissen G, Otsuka K, Halberg F, Consonni D, Nicolini U. Circadian rhythm of maternal blood pressure and fetal growth. Biomed Pharmacother 2005; 59(1): S86-S91.

[42] Murphy VE, Smith R, Giles WB, Clifton VL. Endocrine Regulation of Human Fetal Growth: The Role of the Mother, Placenta, and Fetus. Endocr Rev 2006; 27(2): 141-69. Available from: http: //edrv.endojournals.org/cgi/content/abstract/27/2/141

[43] Hornberger LK, Sahn DJ. Rhythm abnormalities of the fetus. Heart 2007; 93(10): 1294-300. Available from: http: //dx.doi.org/ $10.1136 /$ hrt.2005.069369
[44] van Leeuwen P, Lange S, Bettermann H, Grönemeyer D, Hatzmann W. Fetal heart rate variability and complexity in the course of pregnancy. Early Hum Dev 1999; 54(3): 259-69.

[45] Wakai RT. Assessment of fetal neurodevelopment via fetal magnetocardiography. Exp Neurol 2004; 190 (Suppl 1): S65-S71. Available from: http: //dx.doi.org/10.1016/j.expneurol.2004.04.019

[46] Muro M, Shono H, Shono M, Uchiyama A, Iwasaka T. Changes in diurnal variations in the fetal heart rate baseline with advancing gestational age. Sleep Biol Rhythms 2004; 2: 1: 83-5.

[47] Lunshof S, Boer K, Wolf H, van Hoffen G, Bayram N, Mirmiran $\mathrm{M}$. Fetal and maternal diurnal rhythms during the third trimester of normal pregnancy: Outcomes of computerized analysis of continuous twenty-four-hour fetal heart rate recordings. Am J Obstet Gynecol 1998; 178: 247U-254.

[48] Shono H, Muro M, Kohara M, Ito Y, Nagasawa T, Sugimori H. Fetal heart rate recorder for long-duration use in active full-term pregnant women. Obstet Gynecol 1994; 83: 301-6.

[49] Greene KR, Rosen KG. Long-term ST waveform changes in the ovine fetal electrocardiogram: the relationship to spontaneous labour and intrauterine death. Clin Phys Physiol Meas 1989; 10(4B): 33-40. Available from: http: //stacks.iop.org/0143-0815/10/33

[50] Rosen K, Dagbjartsson A, Henriksson B, Lagercrantz H, Kjellmer I. The relationship between circulating catecholamines and ST waveform in the fetal lamb electrocardiogram during hypoxia 3. Am J Obstet Gynecol 1984; 149: 190-5.

[51] Westgate J, Bennet L, Brabyn C, Williams C, Gunn A. ST waveform changes during repeated umbilical cord occlusions in nearterm fetal sheep. Am J Obstet Gynecol 2001; 184: 743-51.

[52] Westgate J, Harris M, Curnow JSH, Greene KR. Randomised trial of cardiotocography alone or with ST waveform analysis for intrapartum monitoring. Lancet 1992; 340(8813): 194-8. Available from: http: //www.sciencedirect.com/science/article/B6T1B-49K5G 3B-7H/2/821ed8d2f7a9e29f6c095caeae5df4ea

[53] Amer-Wahlin I, Hellsten C, Noren H, et al. Cardiotocography only versus cardiotocography plus ST analysis of fetal electrocardiogram for intrapartum fetal monitoring: a Swedish randomised controlled trial. Lancet 2001; 358: 534-8.

[54] Kwee A, van der Hoorn-van den Beld CW, Veerman J, Dekkers A, Visser G. STAN S21 fetal heart monitor for fetal surveillance during labor: an observational study in 637 patients. J Matern Fetal Neonatal Med 2004; 15: 400-7.

[55] Amer-Wahlin I, Kallen K, Herbst A, Rydhstroem H, Sundstrom A, Marsal K. Implementation of new medical techniques: experience from the Swedish randomized controlled trial on fetal ECG during labor. J Matern Fetal Neonatal Med 2005; 18: 93-100.

[56] Westerhuis M, Moons K, van Beek E, et al. A randomised clinical trial on cardiotocography plus fetal blood sampling versus cardiotocography plus ST-analysis of the fetal electrocardiogram (STAN(R)) for intrapartum monitoring. BMC Pregnancy Childbirth 2007; 7(1): 13. Available from: http: //www.biomedcentral. com/1471-2393/7/13

[57] Outram NJ, Ifeachor EC, Eetvelt PWJV, Curnow JSH. Techniques for optimal enhancement and feature extraction of fetal electrocardiogram. In: IEE Proc.-Sci. Meas Technol 1995; 142: 482-9.

[58] Genevier ESG, Deans AC, Carter MC, Steer PJ. Separation of fetal and maternal ECG complexes from a mixed signal using an algorithm based on linear regression. Med Eng Phys 1995; 17(7): 51422.

[59] Lai KC, Shynk JJ. A successive cancellation algorithm for fetal heart-rate estimation using an intrauterine ECG signal. IEEE Trans Biomed Eng 2002; 49(9): 943-54.

[60] De Moor B. Database for the Identification of Systems (DaISy) 1997. Available from: http: //homes.esat.kuleuven.be/ smc/daisy/

[61] Goldberger AL, Amaral LAN, Glass L, et al. PhysioBank, PhysioToolkit, and PhysioNet: Components of a New Research Resource for Complex Physiologic Signals. Circulation 2000; 101(23): e215-e220. Circulation Electronic Pages: http: //circ. ahajournals.org/cgi/content/full/101/23/e215

[62] Wladimiroff JW, Pilu G, Ed. Ultrasound and the Fetal Heart. New. York, NY: Parthenon Publishing 1996.

[63] Drose JA. Fetal Echocardiography. Saunders W B Co 1998

[64] Kovacs F, Torok M, Habermajer I. A rule-based phonocardiographic method for long-term fetal heart rate monitoring. IEEE Trans Biomed Eng 2000; 47(1): 124-30. 
[65] Várady P, Wildt L, Benyó Z, Hein A. An advanced method in fetal phonocardiography. Comput Methods Prog Biomed 2003; 71(3): 283-96.

[66] CancerWEB; 1998. Available from: http: //cancerweb.ncl.ac.uk/.

[67] Phonocardiography 2008. Available from: http: //www.britannica. com/

[68] Bloom SL, Spong CY, Thom E, et al. Fetal Pulse Oximetry and Cesarean Delivery. N Engl J Med 2006; 355(21): 2195-202. For the National Institute of Child Health and Human Development Maternal-Fetal Medicine Units Network. Available from: http: //content.nejm.org/cgi/content/abstract/355/21/2195

[69] Signorini MG, Magenes G, Cerutti S, Arduini D. Linear and nonlinear parameters for the analysis of fetal heart rate signal from cardiotocographic recordings. IEEE Trans Biomed Eng 2003; 50(3): 365-74. Available from: http: //dx.doi.org/10.1109/TBME. 2003.808824

[70] van Geijn HP. Developments in CTG analysis. Baillieres Clin Obstet Gynaecol 1996; 10(2): 185-209.

[71] Kariniemi V, Hukkinen K. Quantification of fetal heart rate variability by magnetocardiography and direct electrocardiography. Am J Obstet Gynecol 1977; 128(5): 526-30.

[72] Chen M, Wakai RT, Veen BV. Eigenvector based spatial filtering of fetal biomagnetic signals. J Perinat Med 2001; 29(6): 486-96.

[73] ter Brake HJM, Rijpma AP, Stinstra JG, et al. Fetal magnetocardiography: clinical relevance and feasibility. Physica C 2002; 368: 10-17. Available from: http: //www.ingentaconnect.com/content/ els/09214534/2002/00000368/00000001/art01132

[74] Wakai RT, Lutter WJ. Matched-filter template generation via spatial filtering: application to fetal biomagnetic recordings. IEEE Trans Biomed Eng 2002; 49(10): 1214-7

[75] Kautz RL. Jim Zimmerman and the SQUID. IEEE Trans Appl Supercond 2001; 11(1): 1026-31.

[76] Larks SD. Present Status of Fetal Electrocardiography. IEEE Trans Biomed Eng 1962; 9(3): 176-80.

[77] Park YC, Lee KY, Youn DH, Kim NH, Kim WK, Park SH. On detecting the presence of fetal R-wave using the moving averaged magnitude difference algorithm. IEEE Trans Biomed Eng 1992; 39(8): 868-71.

[78] Shao M, Barner KE, Goodman MH. An interference cancellation algorithm for noninvasive extraction of transabdominal fetal electroencephalogram (TaFEEG). IEEE Trans Biomed Eng 2004; 51(3): 471-83.

[79] Martens SMM, Rabotti C, Mischi M, Sluijter RJ. A robust fetal ECG detection method for abdominal recordings. Physiol Meas 2007; 28(4): 373-88. Available from: http: //dx.doi.org/10.1088/ 0967-3334/28/4/004

[80] Sameni R. Extraction of Fetal Cardiac Signals from an Array of Maternal Abdominal Recordings. Sharif University of Technology Institut National Polytechnique de Grenoble 2008. Available from: http: //www.sameni.info/Publications/Thesis/PhDThesis.pdf.

[81] Sameni R, Shamsollahi MB, Jutten C, Clifford GD. A Nonlinear Bayesian Filtering Framework for ECG Denoising. IEEE Trans Biomed Eng 2007; 54(12): 2172-85.

[82] Sameni R, Shamsollahi MB, Jutten C. Model-based Bayesian filtering of cardiac contaminants from biomedical recordings. Physiol Meas 2008; 29(5): 595-613.

[83] Li C, Zheng C, Tai C. Detection of ECG characteristic points using wavelet transforms. IEEE Trans Biomed Eng 1995; 42(1): 21-8.

[84] Khamene A, Negahdaripour S. A new method for the extraction of fetal ECG from the composite abdominal signal. IEEE Trans Biomed Eng 2000; 47(4): 507-16.

[85] Akay M, Akay M, Mulder E. Examining fetal heart-rate variability using matching pursuits. IEEE Eng Med Biol Mag 1996; 15(5): 64-7.

[86] Damen AA, Van Der Kam J. The use of the singular value decomposition in electrocardiography. Med Biol Eng Comput 1982; 20(4): 473-82.

[87] Vanderschoot J, Callaerts D, Sansen W, Vandewalle J, Vantrappen G, Janssens J. Two methods for optimal MECG elimination and FECG detection from skin electrode signals. IEEE Trans Biomed Eng 1987; BME-34: 233-43.

[88] Callaerts D. Signal separation methods based on singular value decomposition and their application to the real-time extraction of the fetal electrocardiogram from cutaneous recordings. K.U.Leuven $\mathrm{PhD}$ thesis 1989.
[89] Kanjilal PP, Palit S, Saha G. Fetal ECG extraction from singlechannel maternal ECG using singular value decomposition. IEEE Trans Biomed Eng 1997; 44: 51-9.

[90] Zarzoso V, Nandi AK, Bacharakis E. Maternal and fetal ECG separation using blind source separation methods. IMA J Math Appl Med Biol 1997; 14: 207-25.

[91] Zarzoso V, Nandi AK. Comparison between blind separation and adaptive noise cancellation techniques for fetal electrocardiogram extraction. In: IEE Colloquium on. Medical Applications for Signal Processing 1999. p. 1/1-1/6.

[92] Zarzoso V, Nandi AK. Noninvasive fetal electrocardiogram extraction: blind separation versus adaptive noise cancellation. IEEE Trans Biomed Eng 2001; 48(1): 12-8.

[93] Cardoso JF. Multidimensional independent component analysis. In: Proceedings of the IEEE International Conference on Acoustics, Speech, and Signal Processing (ICASSP '98) 1998; 4: 1941-1944.

[94] De Lathauwer L, De Moor B, Vandewalle J. Fetal electrocardiogram extraction by blind source subspace separation. IEEE Trans Biomed Eng 2000; 47: 567-72.

[95] Barros AK, Cichocki A. Extraction of specific signals with temporal structure. Neural Comput 2001; 13(9): 1995-2003. Available from: http: //dx.doi.org/10.1162/089976601750399272

[96] Zhang ZL, Yi Z. Extraction of temporally correlated sources with its application to non-invasive fetal electrocardiogram extraction. Neurocomputing 2006; 69(7-9): 894-9.

[97] Li Y, Yi Z. An algorithm for extracting fetal electrocardiogram. Neurocomput 2008; 71(7-9): 1538-42.

[98] Vigneron V, Paraschiv-Ionescu A, Azancot A, Sibony O, Jutten C. Fetal electrocardiogram extraction based on non-stationary ICA and wavelet denoising. Signal Processing and Its Applications, Proceedings Seventh International Symposium on 2003; 2: 69-72.

[99] Jafari MG, Chambers JA. Fetal electrocardiogram extraction by sequential source separation in the wavelet domain. IEEE Trans Biomed Eng 2005; 52(3): 390-400. Available from: http: //dx.doi. org/10.1109/TBME.2004.842958

[100] Sameni R, Jutten C, Shamsollahi MB. Multichannel Electrocardiogram Decomposition Using Periodic Component Analysis. IEEE Trans Biomed Eng 2008; 55(8): 1935-40.

[101] Sameni R, Jutten C, Shamsollahi MB. A Deflation Procedure for Subspace Decomposition. IEEE Trans Signal Process 2010; 58(4): 2363-74.

[102] Schreiber T, Kaplan DT. Signal separation by nonlinear projections: The fetal electrocardiogram. Phys Rev E 1996; 53: R4326-R29.

[103] Schreiber T, Kaplan DT. Nonlinear noise reduction for electrocardiograms. Chaos 1996; 6(1): 87-92.

[104] Richter M, Schreiber T, Kaplan DT. Fetal ECG extraction with nonlinear state-space projections. IEEE Trans Biomed Eng 1998; 45(1): 133-7.

[105] Kantz H, Schreiber T. Human ECG: nonlinear deterministic versus stochastic aspects. Meas Sci Technol, IEEE Proc 1998; 145: 27984 .

[106] Kotas M. Projective filtering of time-aligned ECG beats. IEEE Trans Biomed Eng 2004; 51(7): 1129-39.

[107] Geselowitz DB. On the theory of the electrocardiogram. Proc IEEE 1989; 77: 857-76.

[108] Gulrajani RM. The forward and inverse problems of electrocardiography. Eng Med Biol Mag IEEE 1998; 17(5): 84-101.

[109] Oostendorp TF, van Oosterom A, Jongsma HW. The fetal ECG throughout the second half of gestation. Clin Phys physiol Meas 1989; 10(2): 147-60.

[110] Oostendorp TF, van Oosterom A, Jongsma HW. The effect of changes in the conductive medium on the fetal ECG throughout gestation. Clin Phys Physiol Meas 1989; 10(Sup. B): 11-20.

[111] Dössel O. Inverse problem of electro- and magnetocardiography: Review and recent progress. Int J Bioelectromagnetism 2000; 2(2).

[112] van Oosterom A. Beyond the dipole; modeling the genesis of the electrocardiogram. 100 years einthoven. Leiden: The Einthoven Foundation 2002; pp. 7-15.

[113] Malmivuo JA, Plonsey R. Bioelectromagnetism, principles and applications of bioelectric and biomagnetic fields. oxford: University Pres 1995. Available from: http: //butler.cc.tut.fi/ malmivuo/bem/ bembook

[114] Frank E. An Accurate, Clinically Practical System For Spatial Vectorcardiography. Circulation 1956; 13(5): 737-749. Available from: http: //circ.ahajournals.org/cgi/content/abstract/13/5/737 
[115] Fletcher GF, Balady G, Froelicher VF, Hartley LH, Haskell WL, Pollock ML. Exercise Standards: A Statement for Healthcare Professionals From the American Heart Association. Circulation 2001; 91(2): 580-615.

[116] Geselowitz DB. On bioelectric potentials in an inhomogeneous volume conductor. Biophys J 1967; 7: 1-11.

[117] McSharry PE, Clifford GD, Tarassenko L, Smith LA. A Dynamic Model for Generating Synthetic Electrocardiogram Signals. IEEE Trans Biomed Eng 2003; 50: 289-94.

[118] Clifford GD, McSharry PE. A realistic coupled nonlinear artificial $\mathrm{ECG}, \mathrm{BP}$, and respiratory signal generator for assessing noise performance of biomedical signal processing algorithms. Proceeding of SPIE International Symposium on Fluctuations and Noise 2004; 5467(34): 290-301.

[119] Sameni R, Shamsollahi MB, Jutten C, Babaie-Zadeh M. Filtering Noisy ECG Signals Using the Extended Kalman Filter Based on a Modified Dynamic ECG Model. In: Proceedings of the $32^{\text {nd }}$ Annual International Conference on Computers in Cardiology. Lyon: France 2005; 1017-20.

[120] Clifford GD. A Novel Framework for Signal Representation and Source Separation. J Biol Sys 2006; 14(2): 169-83.
[121] Sameni R, Clifford GD, Jutten C, Shamsollahi MB. Multichannel ECG and Noise Modeling: Application to Maternal and Fetal ECG Signals. EURASIP J Adv Signal Process 2007; Article ID 43407, 14 pages. ISSN 1687-6172, doi: 10.1155/2007/43407. Available from: http: //www.hindawi.com/GetArticle.aspx?doi=10.1155/ 2007/43407

[122] Sameni R, Vrins F, Parmentier F, et al. Electrode Selection for Noninvasive Fetal Electrocardiogram Extraction using Mutual Information Criteria. In: Proceeding. Of the $26^{\text {th }}$ International Workshop on Bayesian Inference and Maximum Entropy Methods in Science and Engineering (MaxEnt 2006). vol. 872. CNRS, Paris, France 2006; p. 97-104.

[123] Li Q, Mark RG, Clifford GD. Robust heart rate estimation from multiple asynchronous noisy sources using signal quality indices and a Kalman Filter. IOP Physiol Meas 2008; 29(1): 15-32.

[124] Li Q, Mark RG, Clifford GD. Artificial arterial blood pressure artifact models and an evaluation of a robust blood pressure and heart rate estimator. BioMed Eng OnLine 2009; 8(1): 13. Available from: http: //www.biomedical-engineering-online.com/ content/8/1/13

(C) Sameni and Clifford; Licensee Bentham Open.

This is an open access article licensed under the terms of the Creative Commons Attribution Non-Commercial License (http://creativecommons.org/licenses/ by-nc/3.0/) which permits unrestricted, non-commercial use, distribution and reproduction in any medium, provided the work is properly cited. 